\title{
Mental health outcomes in HIV and childhood maltreatment: a systematic review
}

\author{
Georgina Spies ${ }^{1}$, Tracie O Afifi ${ }^{4}$, Sarah L Archibald ${ }^{5,6}$, Christine Fennema-Notestine ${ }^{5,6}$, Jitender Sareen ${ }^{3}$ \\ and Soraya Seedat ${ }^{1,2^{*}}$
}

\begin{abstract}
Background: High rates of childhood maltreatment have been documented in HIV-positive men and women. In addition, mental disorders are highly prevalent in both HIV-infected individuals and victims of childhood maltreatment. However, there is a paucity of research investigating the mental health outcomes associated with childhood maltreatment in the context of HIV infection. The present systematic review assessed mental health outcomes in HIV-positive individuals who were victims of childhood maltreatment.

Methods: A systematic search of all retrospective, prospective, or clinical trial studies assessing mental health outcomes associated with HIV and childhood maltreatment. The following online databases were searched on 25-31 August 2010: PubMed, Social Science Citation Index, and the Cochrane Library (the Cochrane Central Register of Controlled Trials and the Cochrane Developmental, Psychosocial and Learning Problems, HIV/AIDS, and Depression, Anxiety and Neurosis registers).

Results: We identified 34 studies suitable for inclusion. A total of 14,935 participants were included in these studies. A variety of mixed mental health outcomes were reported. The most commonly reported psychiatric disorders among HIV-positive individuals with a history of childhood maltreatment included: substance abuse, major depressive disorder, and posttraumatic stress disorder. An association between childhood maltreatment and poor adherence to antiretroviral regimens was also reported in some studies.

Conclusion: A broad range of adult psychopathology has been reported in studies of HIV-infected individuals with a history of childhood maltreatment. However, a direct causal link cannot be well established. Longer term assessment will better delineate the nature, severity, and temporal relationship of childhood maltreatment to mental health outcomes.
\end{abstract}

Keywords: AIDS, Anxiety, Childhood maltreatment, Depression, HIV, Psychiatric morbidity, Substance abuse

\section{Background}

Abuse is a common phenomenon in countries where the prevalence rate of HIV is also high and can include physical, sexual and emotional violence and deprivation or neglect [1]. Studies conducted in developing countries such as South Africa and other African countries have reported high rates of abuse in both adults and children.

\footnotetext{
* Correspondence: sseedat@sun.ac.za

'South African Research Chairs Initiative (SARChl), PTSD program, Francie van Zijl drive, Department of Psychiatry, University of Stellenbosch, Cape Town 7505, South Africa

${ }^{2}$ MRC Unit on Anxiety and Stress Disorders, Francie van Zijl drive,

Department of Psychiatry, University of Stellenbosch, Cape Town 7505, South Africa

Full list of author information is available at the end of the article
}

This includes intimate partner violence (IPV), rape, and childhood abuse or maltreatment [1-3]. Childhood maltreatment has been defined in many different ways. However, for the present review, childhood maltreatment included emotional, physical, and sexual abuse and emotional and physical neglect. According to Bernstein et al. [4] sexual abuse is defined as 'sexual contact or conduct between a child younger than 18 years of age and an adult or older person.' Physical abuse is defined as 'bodily assaults on a child by an adult or older person that posed a risk of or resulted in injury.' Emotional abuse is defined as 'verbal assaults on a child's sense of worth or well-being or any humiliating or demeaning behaviour directed toward a child by an adult or older

\section{Biomed Central}

(c) 2012 Spies et al.; licensee BioMed Central Ltd. This is an Open Access article distributed under the terms of the Creative Commons Attribution License (http://creativecommons.org/licenses/by/2.0), which permits unrestricted use, distribution, and reproduction in any medium, provided the original work is properly cited. 
person.' Physical neglect is defined as 'the failure of caretakers to provide for a child's basic physical needs, including food, shelter, clothing, safety, and health care.' Emotional neglect is defined as 'the failure of caretakers to meet children's basic emotional and psychological needs, including love, belonging, nurturance, and support' [4]. Although women are more vulnerable and regarded as particularly at risk for abuse, men are also victims of rape and childhood maltreatment.

Many studies have investigated the link between adverse childhood experiences such as physical and/or sexual abuse and HIV risk. The experience of childhood maltreatment may increase HIV infection risk indirectly by increasing high-risk behaviors or by interfering with HIV prevention choices [5]. For example, many of the outcomes associated with childhood maltreatment place individuals at increased risk of contracting HIV through behaviors such as transactional sex, unprotected sex, inability to negotiate condom use, alcohol and/or drug abuse, early onset of sexual activities, and multiple sex partners [6-10]. In addition, childhood maltreatment may directly increase the risk of HIV infection through sexual abuse. Injury and the tearing of tissue resulting from sexual violence may increase the likelihood of HIV infection [11]. Studies have also found that childhood maltreatment is strongly associated with adult revictimization which can further increase the risk for HIV among women [5].

The mental health outcomes of HIV-infected individuals have been well documented to date. Research suggests a significant burden of mental illness in individuals living with HIV/AIDS, both globally and in the developing world. Mental illnesses documented in HIVinfected individuals include predominantly substance use, anxiety, and mood disorders [12-19]. Moreover, it has been suggested that HIV disease progression may be hastened by mental disorders such as depression and anxiety [20].

Similarly, research suggests the long-term mental health outcomes of childhood maltreatment include predominantly substance, anxiety, and mood disorders [21,22]. Interestingly, Kaplow and Widom [23] followed 496 individuals with neglect, physical and sexual abuse prior to the age of 12 into adulthood. Their research suggests that an earlier onset of maltreatment predicted more symptoms of anxiety and depression in adulthood, while controlling for gender, race, current age and reports of other abuse. Later onset of maltreatment was predictive of more behavioral problems in adulthood [23]. In a review of child sexual abuse, Johnson [11] outlines a number of child and adult psychological and behavioral consequences of child sexual abuse. These include substance use disorders, and anxiety and mood disorders, amongst others [11].
Although many studies have focused on mental health outcomes in childhood maltreatment and HIV separately, there is a paucity of research investigating childhood maltreatment and HIV in combination, and the associated mental health outcomes in dually affected men and women. HIV-infected women may face more current and past negative life events than men in developing parts of the world [13] and this may lead to significant adult psychopathology and poor adherence to antiretroviral medications [24,25]. In light of this, it is evident that HIV-positive individuals, women in particular, are vulnerable to risk factors associated with abuse, and abuse-related changes in behavioral functioning, which may complicate HIV infection. A systematic assessment and summary of the available evidence is therefore warranted in order to add to the available evidence for both clinical and research decision making.

\section{Methods}

\section{Search strategy and selection criteria}

We searched the electronic databases PubMed, Social Science Citation Index, the Cochrane Library (The Cochrane Central Register of Controlled Trials: CENTRAL) and the Cochrane Developmental, Psychosocial and Learning Problems, HIV/AIDS, and Depression, Anxiety and Neurosis registers on 25-30 August 2010. No limit on the time period was applied to the search in order to avoid omission of relevant studies. Reference lists of articles identified through database searches and bibliographies of systematic and non-systematic review articles were examined to identify further relevant studies. We included all English language, original research (retrospective and prospective studies) and clinical trials reporting mental health outcomes of childhood trauma in HIV-positive individuals. The population included adult men and women already infected with HIV/AIDS who experienced childhood maltreatment prior to 18 years of age. We excluded systematic and non-systematic review articles and studies of no direct relevance to the comprehensive search. The PubMed search included the following terms: childhood abuse AND HIV. The full search details are as follows: (('childhood'[Journal] OR 'childhood'[All Fields]) AND ('substance-related disorders'[MeSH Terms] OR ('substance-related'[All Fields] AND 'disorders'[All Fields]) OR 'substance-related disorders'[All Fields] OR 'abuse'[All Fields])) AND ('hiv'[MeSH Terms] OR 'hiv'[All Fields]). No filters were included to ensure that all relevant papers were retrieved. The PubMed search selected those studies that addressed childhood abuse and HIV in all fields. An initial search of titles was undertaken by the reviewer (GS). Studies were included irrespective of sample size and period of follow-up. Titles and abstracts of studies that appeared relevant were then assessed to determine whether they met the inclusion criteria. Abstracts that did not meet the inclusion 
criteria were rejected. The reviewer assessed full texts of articles that appeared to meet the inclusion criteria of the present study. Information was extracted regarding population characteristics and sample size, study design, outcomes measured and results. No exploration of publication bias was undertaken and it was not possible to conduct a sensitivity analysis for the current review article due to the fact that no meta-analyses were conducted (see Figure 1).

\section{Results}

All databases searched yielded abstracts, and there were duplicates between the databases. All the studies had published results in peer-reviewed journals. Two hundred and five abstracts were identified and reviewed. Of the 205 abstracts identified, 171 articles were excluded. Studies were excluded if they were: of no relevance to the present review, systematic or non-systematic review articles, or not conducted in the population of interest. Titles and abstracts of 52 studies that appeared relevant were then assessed to determine whether they met the inclusion criteria. Of the relevant studies reviewed, 34 articles met inclusion criteria. Three of the 34 articles were sourced from reference lists of other manuscripts. Full text articles for all 34 studies were accessed and reviewed. The reviewed articles are summarized in Table 1 . There was heterogeneity in sample characteristics, study methodologies and outcome measures among all studies reviewed in this article. Moreover, some studies included an HIV comparison group whereas others did not, further limiting comparability. For these reasons, it was decided that a meta-analysis of these data was not feasible. Variability in measurement of mental health impairment was noted. Psychiatric symptoms and disorders were assessed according to standard diagnostic criteria, using a structured clinician administered interview and/or through self-report (see Table 1). Although some studies differentiated symptoms and diagnoses, others reported more global levels of psychological distress. For example, two articles sourced reported on global psychological distress and mental health in general, without delineating whether symptoms were depressive in nature or anxiety related, for example [26,27]. Furthermore, some studies simply stated the percentage of HIV-positive maltreatment victims reporting symptoms of anxiety. Although these studies reported global anxiety levels, they failed to differentiate by diagnosis [7,28-30].

A history of childhood maltreatment was also assessed in different ways, but all studies relied on self-reported history of childhood maltreatment, and most assessments were retrospective in nature. In some studies, childhood maltreatment included various forms/types such as physical abuse and neglect, emotional abuse and neglect, and sexual abuse [43]. Other studies only examined childhood sexual abuse (CSA) $[6,26,40]$ or combined sexual and physical abuse into one category of child abuse $[25,39,41]$. Some studies utilized validated self-report measures sensitive in tapping into various forms of childhood abuse and neglect $[38,43]$. A widely used example of such a measure is the Childhood

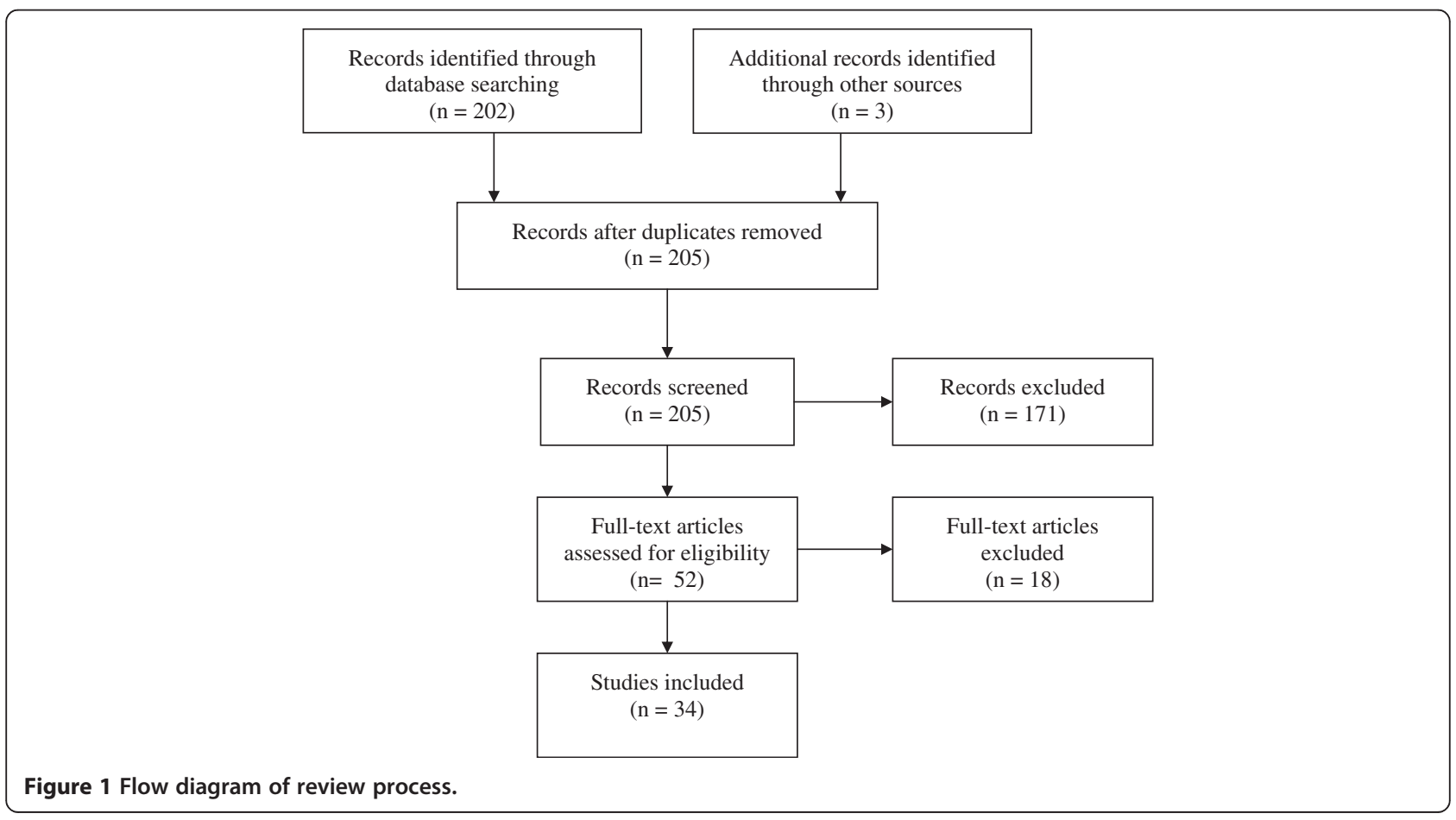


Table 1 Summary of $\mathbf{3 4}$ articles selected for review

\begin{tabular}{|c|c|c|c|c|c|c|c|c|c|c|}
\hline $\begin{array}{l}\text { First } \\
\text { author } \\
\text { [Reference } \\
\text { number] }\end{array}$ & $\begin{array}{l}\text { N (HIV } \\
+/-)\end{array}$ & $\begin{array}{l}\text { Setting } \\
\text { and main } \\
\text { characteristics } \\
\text { of population }\end{array}$ & $\begin{array}{l}\text { Type of } \\
\text { study }\end{array}$ & $\begin{array}{l}\text { Study } \\
\text { design }\end{array}$ & $\begin{array}{l}\text { Methods } \\
\text { (measurement of } \\
\text { exposure and } \\
\text { outcomes) }\end{array}$ & $\begin{array}{l}\text { Summary of } \\
\text { outcomes }\end{array}$ & $\begin{array}{l}\text { Childhood } \\
\text { maltreatment }\end{array}$ & $\begin{array}{l}\text { Mental health } \\
\text { outcomes }\end{array}$ & $\begin{array}{l}\text { High-risk } \\
\text { behaviors and } \\
\text { revictimization }\end{array}$ & $\begin{array}{l}\text { Treatment } \\
\text { adherence }\end{array}$ \\
\hline $\begin{array}{l}\text { (1) Masten } \\
\text { (2007) [31] }\end{array}$ & $\begin{array}{l}49 \\
(49 / 0)\end{array}$ & $\begin{array}{l}\text { USA.49 bisexual } \\
\text { HIV-positive } \\
\text { men with } \\
\text { childhood } \\
\text { sexual abuse } \\
\text { (CSA) histories. } \\
\text { The majority } \\
\text { were African- } \\
\text { American } \\
\text { men. }\end{array}$ & $\begin{array}{l}\text { Intervention } \\
\text { study. }\end{array}$ & $\begin{array}{l}\text { Baseline } \\
\text { survey } \\
\text { for a coping } \\
\text { group } \\
\text { intervention } \\
\text { trial. }\end{array}$ & $\begin{array}{l}\text { Participants were } \\
\text { individually screened } \\
\text { at baseline using a } \\
\text { structured clinical } \\
\text { interview assessing: } \\
\text { demographics, sexual } \\
\text { abuse history, } \\
\text { depression, } \\
\text { posttraumatic stress } \\
\text { and risk to self or } \\
\text { others. }\end{array}$ & $\begin{array}{l}\text { (1) Full criteria } \\
\text { for posttraumatic } \\
\text { stress disorder } \\
\text { (PTSD). }\end{array}$ & $\begin{array}{l}\text { All participants } \\
\text { reported some } \\
\text { form of sexual } \\
\text { abuse history } \\
\text { before the age } \\
\text { of } 18 \text {, with } \\
90 \% \text { reporting } \\
\text { unwanted } \\
\text { penetrative } \\
\text { anal sex. The } \\
\text { average } \\
\text { participant age } \\
\text { at first abuse } \\
\text { was } 8.9 \text { years. } \\
\text { Most reported } \\
\text { more than one } \\
\text { abusive } \\
\text { experience and } \\
\text { frequently } \\
\text { had a } \\
\text { prolonged } \\
\text { abuse exposure. }\end{array}$ & $\begin{array}{l}\text { A total of } 21 \\
\text { men }(42.9 \%) \\
\text { met criteria } \\
\text { for PTSD. }\end{array}$ & & \\
\hline $\begin{array}{l}\text { (2) Mimiaga } \\
\text { (2009) [8] }\end{array}$ & $\begin{array}{l}4295 \\
(258 / \\
4037)\end{array}$ & $\begin{array}{l}\text { USA. } 4295 \text { men } \\
\text { who have } \\
\text { sex with } \\
\text { men (MSM) } \\
\text { enrolled in } \\
\text { the EXPLORE } \\
\text { study. }\end{array}$ & $\begin{array}{l}\text { Intervention } \\
\text { study. }\end{array}$ & $\begin{array}{l}\text { Longitudinal } \\
\text { research } \\
\text { design. } \\
\text { Intervention } \\
\text { lasted } 48 \\
\text { months with } \\
\text { assessments } \\
\text { every } \\
6 \text { months. }\end{array}$ & $\begin{array}{l}\text { HIV infection was } \\
\text { the primary efficacy } \\
\text { outcome. Abuse } \\
\text { histories, drug and } \\
\text { alcohol use and } \\
\text { other psychosocial } \\
\text { factors were assessed. } \\
\text { A shortened version } \\
\text { of the Center for } \\
\text { Epidemiologic Studies } \\
\text { Depression Scale } \\
\text { (CES-D) assessed } \\
\text { depression. }\end{array}$ & $\begin{array}{l}\text { (1) Depression. } \\
\text { (2) Drug use. }\end{array}$ & $\begin{array}{l}39.7 \% \text { had a } \\
\text { history of CSA. }\end{array}$ & $\begin{array}{l}\text { Participants } \\
\text { with a history } \\
\text { of CSA were } \\
\text { at increased } \\
\text { risk for HIV } \\
\text { infection over } \\
\text { study follow-up. } \\
\text { Among } \\
\text { participants } \\
\text { reporting CSA, } \\
\text { the EXPLORE } \\
\text { intervention had } \\
\text { no effect in } \\
\text { reducing HIV } \\
\text { infection rates. } \\
\text { Participants } \\
\text { reporting CSA } \\
\text { were significantly } \\
\text { more likely to } \\
\text { have symptoms } \\
\text { of depression } \\
\text { and use } \\
\text { nonprescription } \\
\text { drugs. }\end{array}$ & & \\
\hline
\end{tabular}


Table 1 Summary of $\mathbf{3 4}$ articles selected for review (Continued)

\begin{tabular}{|c|c|c|c|c|c|c|c|c|}
\hline & $\begin{array}{l}247 \\
(247 / 0) .\end{array}$ & $\begin{array}{l}\text { USA. } 130 \text { women } \\
\text { and } 117 \text { men } \\
\text { with a } \\
\text { history of } \\
\text { CSA. All } \\
\text { men reported } \\
\text { having sex } \\
\text { with men. }\end{array}$ & $\begin{array}{l}\text { Intervention } \\
\text { study. }\end{array}$ & $\begin{array}{l}\text { Randomized } \\
\text { controlled } \\
\text { behavioral } \\
\text { intervention } \\
\text { trial with } \\
12 \text {-month } \\
\text { follow-up. }\end{array}$ & $\begin{array}{l}\text { A structured } \\
\text { interview assessed } \\
\text { depression, PTSD, } \\
\text { and risk to self or } \\
\text { others. A modified } \\
\text { and expanded } \\
\text { version of the } \\
\text { Traumatic } \\
\text { Experiences } \\
\text { Questionnaire } \\
\text { (TEQ) assessed } \\
\text { exposure } \\
\text { to traumatic } \\
\text { events, including } \\
\text { sexual abuse } \\
\text { during childhood, } \\
\text { and adulthood. }\end{array}$ & $\begin{array}{l}\text { (1) Sexual } \\
\text { revictimization. } \\
\text { (2) PTSD. }\end{array}$ & $\begin{array}{l}\text { The average age } \\
\text { of first abuse } \\
\text { was } 8.8 \text { years. } \\
\text { Most (90\%) } \\
\text { experienced } \\
\text { penetrative } \\
\text { vaginal or anal } \\
\text { sexual abuse } \\
\text { as a child or } \\
\text { adolescent; } \\
87 \% \text { experienced } \\
\text { sexual } \\
\text { revictimization, } \\
\text { with more than } \\
\text { half of those } \\
\text { revictimized as } \\
\text { children or } \\
\text { adolescents. } \\
\text { Only } 10 \% \text { of } \\
\text { participants } \\
\text { reported a single } \\
\text { episode of abuse. } \\
\text { On average, } \\
\text { CSA lasted } 4 \text { years } \\
\text { and participants } \\
\text { had } 2 \text { abusers } \\
\text { (only } 39 \% \text { of } \\
\text { participants } \\
\text { reported } 1 \\
\text { abuser). }\end{array}$ & $\begin{array}{l}40 \% \text { of the } \\
\text { sample met } \\
\text { diagnostic } \\
\text { criteria for } \\
\text { PTSD. }\end{array}$ \\
\hline $\begin{array}{l}\text { (4) Sikkema } \\
\text { (2004) [33] }\end{array}$ & $\begin{array}{l}28 \\
(28 / 0) .\end{array}$ & $\begin{array}{l}\text { USA. Twenty- } \\
\text { eight } \\
\text { HIV-positive } \\
\text { participants } \\
\text { (7 men } \\
\text { and } 21 \\
\text { women). }\end{array}$ & $\begin{array}{l}\text { Intervention } \\
\text { study. }\end{array}$ & $\begin{array}{l}\text { Baseline } \\
\text { survey } \\
\text { for a coping } \\
\text { intervention } \\
\text { trial. }\end{array}$ & $\begin{array}{l}\text { Trauma Symptom } \\
\text { Checklist: childhood } \\
\text { and adult traumatic } \\
\text { experiences. } \\
\text { Personality } \\
\text { Assessment } \\
\text { Inventory: self- } \\
\text { administered } \\
\text { objective } \\
\text { inventory of adult } \\
\text { personality. Trauma } \\
\text { Symptom Inventory } \\
\text { (TSI): acute and } \\
\text { chronic posttraumatic } \\
\text { symptomatology. }\end{array}$ & $\begin{array}{l}\text { (1) Mood } \\
\text { disorders. } \\
\text { (2) Anxiety } \\
\text { disorders, } \\
\text { including } \\
\text { PTSD. } \\
\text { (3) Substance } \\
\text { abuse. } \\
\text { (4) Personality } \\
\text { disorder. } \\
\text { (5) Revictimization }\end{array}$ & $\begin{array}{l}\text { Prior to age } 12 \text {, } \\
71.4 \% \text { reported } \\
\text { oral sexual } \\
\text { abuse and } \\
85.7 \% \text { reported } \\
\text { penetrative } \\
\text { anal sexual abuse. } \\
\text { During } \\
\text { adolescence, } \\
57.1 \% \text { of the } \\
\text { men } \\
\text { experienced } \\
\text { some form of } \\
\text { sexual abuse. } \\
76.2 \% \text { of } \\
\text { women } \\
\text { reported } \\
\text { unwanted } \\
\text { touching or } \\
\text { fondling, }\end{array}$ & $\begin{array}{l}85 \% \text { of the } \\
\text { participants } \\
\text { received an } \\
\text { indicator of an } \\
\text { Axis I diagnosis: } \\
\text { the most } \\
\text { frequent } \\
\text { diagnostic } \\
\text { categories were } \\
\text { mood disorders } \\
(46.4 \%) \text {, anxiety } \\
\text { disorders, } \\
\text { including PTSD } \\
(32 \%) \text {, and } \\
\text { substance abuse } \\
(25 \%) \text {. On Axis II, } \\
28.5 \% \text { received } \\
\text { at least one } \\
\text { diagnostic } \\
\text { indicator of a }\end{array}$ \\
\hline
\end{tabular}




\begin{tabular}{|c|c|c|c|c|c|c|c|c|}
\hline & & & & & & & $\begin{array}{l}25 \% \text { reported } \\
\text { oral sexual abuse, } \\
\text { and } 57.1 \% \\
\text { reported } \\
\text { penetrative anal } \\
\text { or vaginal sexual } \\
\text { abuse during } \\
\text { childhood } \\
\text { (age } 12 \text { or } \\
\text { younger). During } \\
\text { adolescence, } \\
85.7 \% \text { unwanted } \\
\text { touching or } \\
\text { fondling, } 57.1 \% \\
\text { oral sexual } \\
\text { abuse, and } \\
81.0 \% \\
\text { penetrative } \\
\text { anal or vaginal } \\
\text { sexual abuse. }\end{array}$ & $\begin{array}{l}\text { personality } \\
\text { disorder. }\end{array}$ \\
\hline $\begin{array}{l}\text { (5) Sikkema } \\
\text { (2007) [34] }\end{array}$ & $\begin{array}{l}198 \\
(198 / 0) .\end{array}$ & $\begin{array}{l}\text { USA. } 107 \\
\text { women } \\
\text { and } 91 \text { men } \\
\text { with CSA. } \\
\text { All men } \\
\text { reported } \\
\text { having sex } \\
\text { with men. }\end{array}$ & $\begin{array}{l}\text { Intervention } \\
\text { study. }\end{array}$ & $\begin{array}{l}\text { Baseline } \\
\text { survey for } \\
\text { a coping } \\
\text { intervention } \\
\text { trial. }\end{array}$ & $\begin{array}{l}\text { Depressive } \\
\text { symptomatology: } \\
\text { Beck Depression } \\
\text { Inventory (BDI). } \\
\text { The Impact of } \\
\text { Events Scale: } \\
\text { PTSD symptoms. }\end{array}$ & (1) PTSD. & $\begin{array}{l}89 \% \text { of } \\
\text { participants } \\
\text { experienced } \\
\text { penetrative anal } \\
\text { or vaginal } \\
\text { abuse during } \\
\text { childhood or } \\
\text { adolescence. } \\
\text { Fifty-five percent } \\
\text { of participants } \\
\text { reported sexual } \\
\text { abuse during } \\
\text { both childhood } \\
\text { and adolescence. }\end{array}$ & $\begin{array}{l}40 \% \text { of study } \\
\text { participants } \\
\text { met DSM-IV } \\
\text { diagnostic } \\
\text { criteria for } \\
\text { PTSD. }\end{array}$ \\
\hline $\begin{array}{l}\text { (6) Williams } \\
\text { (2008) [35] }\end{array}$ & $\begin{array}{l}137 \\
(137 / 0) .\end{array}$ & $\begin{array}{l}\text { USA. } 137 \text { HIV- } \\
\text { positive gay } \\
\text { and non-gay } \\
\text { identifying } \\
\text { African- } \\
\text { American and } \\
\text { Latino men } \\
\text { with histories } \\
\text { of CSA. }\end{array}$ & $\begin{array}{l}\text { Intervention } \\
\text { study. }\end{array}$ & $\begin{array}{l}\text { Randomized } \\
\text { clinical } \\
\text { trial compared } \\
\text { the effects of } \\
\text { two 6-session } \\
\text { interventions. }\end{array}$ & $\begin{array}{l}\text { A randomized } \\
\text { clinical trial aimed } \\
\text { at decreasing } \\
\text { high-risk sexual } \\
\text { behaviors, number } \\
\text { of sexual partners, } \\
\text { and depressive } \\
\text { symptoms. The } \\
\text { CES-D assessed } \\
\text { depression. }\end{array}$ & $\begin{array}{l}\text { (1) High levels } \\
\text { of depression } \\
\text { at baseline. } \\
\text { (2) Significant } \\
\text { decrease in } \\
\text { depression from } \\
3 \text { to } 6 \text { months } \\
\text { follow-up. }\end{array}$ & Histories of CSA. & $\begin{array}{l}\text { There were } \\
\text { high levels of } \\
\text { depression at } \\
\text { baseline, } \\
\mathrm{M}=23 \text {. There } \\
\text { was a } \\
\text { significant } \\
\text { decrease in } \\
\text { depressive } \\
\text { symptoms from } \\
\text { the } 3 \text { month } \\
\text { to the } 6 \text { month } \\
\text { follow-up } \\
\text { assessment } \\
\text { for the sample } \\
\text { as a whole }\end{array}$ \\
\hline
\end{tabular}




\begin{tabular}{|c|c|c|c|c|c|c|c|c|}
\hline & & & & & & & & $\begin{array}{l}\text { ( } \mathrm{M}=22.42 \text {, } \\
\text { for } 3 \text { months } \\
\text { depression and } \\
\mathrm{M}=20.66 \text { for } \\
6 \text { months } \\
\text { depression). }\end{array}$ \\
\hline $\begin{array}{l}\text { (7) Holmes } \\
\text { (1997) [36] }\end{array}$ & $\begin{array}{l}95 \\
(95 / 0) .\end{array}$ & $\begin{array}{l}\text { USA. } \\
95 \text { HIV } \\
\text { seropositive } \\
\text { men. The } \\
\text { majority } \\
\text { were Caucasian } \\
\text { men (67\%). } \\
\text { Sexual practices } \\
\text { were homosexual/ } \\
\text { bisexual in } \\
87(92 \%) \\
\text { participants. }\end{array}$ & $\begin{array}{l}\text { Survey } \\
\text { HIV + only }\end{array}$ & $\begin{array}{l}\text { Quantitative } \\
\text { cross-sectional } \\
\text { survey } \\
\text { design. }\end{array}$ & $\begin{array}{l}\text { Sociodemographic } \\
\text { and sexual } \\
\text { abuse histories } \\
\text { were obtained. } \\
\text { The Structured } \\
\text { Clinical Interview } \\
\text { for the DSM } \\
\text { Disorders) was } \\
\text { used to identify } \\
\text { Psychoactive } \\
\text { Substance Use } \\
\text { Disorder (PSUD). }\end{array}$ & $\begin{array}{l}\text { (1) PSUD. } \\
\text { (2) Increased } \\
\text { risk of } \\
\text { intravenous } \\
\text { drug use } \\
\text { (IVDU). }\end{array}$ & $\begin{array}{l}\text { Nineteen } \\
\text { (20\%) participants } \\
\text { had sexual } \\
\text { abuse histories. } \\
\text { First abuse } \\
\text { occurred at a } \\
\text { mean age of } \\
8.1 \text { years. }\end{array}$ & $\begin{array}{l}\text { Fifty-five } \\
\text { (58\%) participants } \\
\text { met the criteria } \\
\text { for a diagnosis } \\
\text { of PSUD at } \\
\text { some time in } \\
\text { their lifetime } \\
\text { and nine (9\%) } \\
\text { currently met } \\
\text { diagnostic criteria. } \\
\text { Men with } \\
\text { histories of } \\
\text { sexual abuse } \\
\text { did not exhibit } \\
\text { a significantly } \\
\text { increased risk of } \\
\text { lifetime or current } \\
\text { PSUD. When rates } \\
\text { were examined } \\
\text { by type of } \\
\text { administration } \\
\text { method, men } \\
\text { with reported } \\
\text { histories of sexual } \\
\text { abuse did show } \\
\text { a significantly } \\
\text { increased risk } \\
\text { of lifetime IVDU. }\end{array}$ \\
\hline $\begin{array}{l}\text { (8) Myers } \\
\text { (2006) [37] }\end{array}$ & $\begin{array}{l}147 \\
(147 / 0) .\end{array}$ & $\begin{array}{l}\text { USA. } 147 \text { HIV- } \\
\text { positive women. } \\
\text { The majority } \\
\text { were African- } \\
\text { American } \\
(n=79) \text {. }\end{array}$ & $\begin{array}{l}\text { Survey } \\
\text { HIV + only }\end{array}$ & $\begin{array}{l}\text { Baseline } \\
\text { survey for } \\
\text { a risk } \\
\text { reduction } \\
\text { intervention } \\
\text { trial. }\end{array}$ & $\begin{array}{l}\text { CSA: revised Wyatt } \\
\text { Sex History } \\
\text { Questionnaire } \\
\text { (WSHQ-R). PTSD: } \\
\text { PTSD diagnostic } \\
\text { module of the } \\
\text { University of } \\
\text { Michigan } \\
\text { version of the } \\
\text { Composite } \\
\text { International } \\
\text { Diagnostic Interview. } \\
\text { Trauma-related } \\
\text { sexual symptoms } \\
\text { were assessed } \\
\text { with the }\end{array}$ & $\begin{array}{l}\text { (1) More PTSD } \\
\text { symptoms in } \\
\text { those abused } \\
\text { by a family } \\
\text { member or by } \\
\text { both a family } \\
\text { and non-family } \\
\text { member. }\end{array}$ & $\begin{array}{l}18 \% \text { of the } \\
\text { women reported } \\
\text { one or more less } \\
\text { severe sexual } \\
\text { abuse incidents. } \\
40 \% \text { experienced } \\
\text { one severe } \\
\text { incident, including } \\
\text { attempted or } \\
\text { completed oral, } \\
\text { anal, or vaginal } \\
\text { sex, and digital } \\
\text { penetration. } \\
18 \% \text { experienced } \\
\text { one severe and } \\
\text { one or more less }\end{array}$ & $\begin{array}{l}\text { The relationship } \\
\text { to perpetrator } \\
\text { was a significant } \\
\text { predictor of } \\
\text { PTSD symptoms, } \\
\text { with more PTSD } \\
\text { symptoms among } \\
\text { those who } \\
\text { reported } \\
\text { intrafamilial } \\
\text { abuse or both } \\
\text { intrafamilial and } \\
\text { extrafamilial abuse } \\
\text { compared with } \\
\text { those who } \\
\text { reported only }\end{array}$ \\
\hline
\end{tabular}


Table 1 Summary of $\mathbf{3 4}$ articles selected for review (Continued)

\begin{tabular}{|c|c|c|c|c|c|c|c|c|}
\hline & & & & & $\begin{array}{l}\text { TSI. Depression } \\
\text { was assessed } \\
\text { with the } \\
\text { CES-D. }\end{array}$ & & $\begin{array}{l}\text { severe incidents, } \\
\text { and } 24 \% \\
\text { experienced two } \\
\text { or more severe } \\
\text { incidents. } 34 \% \text { of } \\
\text { the women } \\
\text { reported being } \\
\text { abused by a } \\
\text { perpetrator that } \\
\text { was not a family } \\
\text { member. } 43 \% \\
\text { reported abuse } \\
\text { by a family member, } \\
\text { and } 24 \% \\
\text { experienced being } \\
\text { abused by both } \\
\text { intra- and } \\
\text { interfamilial } \\
\text { perpetrators. The } \\
\text { mean number of } \\
\text { sexual abuse } \\
\text { incidents was } \\
1.8, \text { with a range } \\
\text { of } 1 \text { to } 6 \text { incidents, } \\
\text { and on average, } \\
\text { the abuse } \\
\text { continued for } \\
2 \text { years. }\end{array}$ & $\begin{array}{l}\text { experiencing } \\
\text { extra familial } \\
\text { abuse. }\end{array}$ \\
\hline $\begin{array}{l}\text { (9) Roy } \\
\text { (2003) [38] }\end{array}$ & $\begin{array}{l}149 \\
(149 / 0) .\end{array}$ & $\begin{array}{l}\text { USA. } 149 \text { HIV } \\
\text { positive } \\
\text { substance- } \\
\text { dependent } \\
\text { patients. There } \\
\text { were more } \\
\text { males than } \\
\text { females in the } \\
\text { sample and } \\
\text { the majority } \\
\text { were African- } \\
\text { American. }\end{array}$ & $\begin{array}{l}\text { Survey } \\
\text { HIV + only }\end{array}$ & $\begin{array}{l}\text { Quantitative } \\
\text { cross-sectional } \\
\text { survey } \\
\text { design. }\end{array}$ & $\begin{array}{l}\text { Structured Clinical } \\
\text { Interview for } \\
\text { DSM-IV: } \\
\text { depression. The } \\
\text { Eysenck } \\
\text { Personality } \\
\text { Questionnaire } \\
\text { (EPQ) assessed } \\
\text { neuroticism, } \\
\text { extraversion, and } \\
\text { psychoticism. The } \\
\text { Childhood Trauma } \\
\text { Questionnaire } \\
\text { (CTQ) assessed for } \\
\text { childhood abuse } \\
\text { and neglect. }\end{array}$ & $\begin{array}{l}\text { (1) Suicidality } \\
\text { (2) Substance } \\
\text { dependence. } \\
\text { (3) Lifetime } \\
\text { depressive } \\
\text { disorder. } \\
\text { 4) High } \\
\text { neuroticism } \\
\text { scores. }\end{array}$ & $\begin{array}{l}\text { HIV positive } \\
\text { patients who } \\
\text { had attempted } \\
\text { suicide had } \\
\text { significantly } \\
\text { higher scores on } \\
\text { the CTQ for } \\
\text { childhood } \\
\text { emotional abuse, } \\
\text { physical abuse, } \\
\text { sexual abuse, } \\
\text { emotional } \\
\text { neglect and } \\
\text { physical neglect. }\end{array}$ & $\begin{array}{l}66(44.3 \%) \text { had } \\
\text { attempted } \\
\text { suicide and } \\
83(55.7 \%) \text { had } \\
\text { not. Significantly } \\
\text { more of those } \\
\text { who had } \\
\text { attempted } \\
\text { suicide were } \\
\text { female. Of the } \\
66 \text { patients, } 51 \\
\text { who had } \\
\text { attempted } \\
\text { suicide had } \\
\text { a primary } \\
\text { substance } \\
\text { dependence } \\
\text { diagnosis. } \\
\text { Significantly } \\
\text { more of the } \\
\text { patients who } \\
\text { had attempted }\end{array}$ \\
\hline
\end{tabular}




\begin{tabular}{|c|c|c|c|c|c|c|c|c|c|}
\hline & & & & & & & & $\begin{array}{l}\text { suicide had a } \\
\text { lifetime history } \\
\text { of a depressive } \\
\text { episode. HIV } \\
\text { positive } \\
\text { attempters also } \\
\text { had significantly } \\
\text { higher } \\
\text { neuroticism } \\
\text { scores on the } \\
\text { EPQ. }\end{array}$ & \\
\hline $\begin{array}{l}10) \text { Allers } \\
(1999)[39]\end{array}$ & $\begin{array}{l}52 \\
(52 / 0) .\end{array}$ & $\begin{array}{l}\text { USA. } 52 \text { HIV- } \\
\text { positive } \\
\text { individuals. } \\
\text { Of the } 45 \\
\text { male and } \\
7 \text { females, } \\
36 \text { were White } \\
\text { and } 16 \text { were } \\
\text { Black. }\end{array}$ & $\begin{array}{l}\text { Survey } \\
\text { HIV + only }\end{array}$ & $\begin{array}{l}\text { Qualitative } \\
\text { survey } \\
\text { design. }\end{array}$ & $\begin{array}{l}\text { A semi-structured } \\
\text { interview conducted } \\
\text { by male HIV } \\
\text { counselors. } \\
\text { This interview } \\
\text { tapped into } \\
\text { variables such as: } \\
\text { history of childhood } \\
\text { abuse, pre-HIV } \\
\text { histories of abusive } \\
\text { or revictimizing } \\
\text { relationships or both, } \\
\text { depression, sexual } \\
\text { compulsivity and } \\
\text { alcohol or other } \\
\text { drug abuse. }\end{array}$ & $\begin{array}{l}\text { (1) History of } \\
\text { alcohol or drug } \\
\text { abuse. } \\
\text { (2) Chronic } \\
\text { depressive } \\
\text { symptomatology. } \\
\text { (3) Revictimization. } \\
\text { (4) Sexually } \\
\text { compulsive } \\
\text { behaviors. }\end{array}$ & $\begin{array}{l}\text { A total of } \\
65 \% \text { ( } n=34) \\
\text { reported a } \\
\text { history of } \\
\text { childhood } \\
\text { sexual or } \\
\text { physical abuse } \\
\text { or both. 35.3\% } \\
\text { ( } n=12 \text { ) } \\
\text { reported } \\
\text { physical abuse } \\
\text { only, and } \\
64.7 \% \text { ( } n=22 \text { ) } \\
\text { reported } \\
\text { sexual abuse. } \\
\text { All } 22 \\
\text { participants } \\
\text { reporting sexual } \\
\text { abuse also } \\
\text { reported } \\
\text { some additional } \\
\text { form of } \\
\text { childhood } \\
\text { physical abuse. } \\
\text { Of these } \\
\text { participants, } \\
88 \% \text { ( } n=30) \\
\text { reported a } \\
\text { history of } \\
\text { alcohol or other } \\
\text { drug abuse, } \\
82 \%(n=28) \\
\text { reported } \\
\text { revictimizing } \\
\text { relationships, } \\
68 \% \text { ( } n=23) \\
\text { reported } \\
\text { chronic depressive } \\
\text { symptomatology, }\end{array}$ & $\begin{array}{l}\text { Of these } \\
\text { participants, } \\
88 \%(n=30) \\
\text { reported a } \\
\text { history of } \\
\text { alcohol or } \\
\text { other drug } \\
\text { abuse, and } 68 \% \\
(n=23) \\
\text { reported } \\
\text { chronic } \\
\text { depressive } \\
\text { symptomatology. }\end{array}$ & $\begin{array}{l}82 \%(n=28) \\
\text { reported } \\
\text { revictimizing } \\
\text { relationships, } \\
\text { and } 50 \% \\
(n=17) \\
\text { reported } \\
\text { engaging in } \\
\text { sexually } \\
\text { compulsive } \\
\text { behaviors. }\end{array}$ \\
\hline
\end{tabular}




\begin{tabular}{|c|c|c|c|c|c|c|c|c|c|}
\hline & & & & & & & $\begin{array}{l}\text { and } 50 \% \\
(n=17) \\
\text { reported } \\
\text { engaging in } \\
\text { sexually } \\
\text { compulsive } \\
\text { behaviors. }\end{array}$ & & \\
\hline $\begin{array}{l}\text { (11) Brennan } \\
\text { (2007) [40] }\end{array}$ & $\begin{array}{l}936 \\
(936 / 0) .\end{array}$ & $\begin{array}{l}\text { USA. } 936 \\
\text { gay and } \\
\text { bisexual men. } \\
\text { The majority } \\
\text { (95.3\%) were } \\
\text { gay and White } \\
(88.8 \%) \text { men. }\end{array}$ & $\begin{array}{l}\text { Survey } \\
H I V+\text { only }\end{array}$ & $\begin{array}{l}\text { Quantitative } \\
\text { cross-sectional } \\
\text { survey } \\
\text { design: } \\
\text { retrospective } \\
\text { data. }\end{array}$ & $\begin{array}{l}\text { A self-administered } \\
\text { survey investigating: } \\
\text { HIV/sexually } \\
\text { transmitted } \\
\text { infection (STI) } \\
\text { status, self-defined } \\
\text { current use of sex- } \\
\text { related drugs, } \\
\text { other HIV risk } \\
\text { behaviors and } \\
\text { history of CSA. }\end{array}$ & $\begin{array}{l}\text { (1) Current } \\
\text { drug abuse. } \\
\text { (2) Transactional } \\
\text { sex practices. }\end{array}$ & $\begin{array}{l}\text { 15.5\% ( } n=134) \\
\text { of survey } \\
\text { respondents } \\
\text { reported a } \\
\text { history of CSA. } \\
\text { Those who } \\
\text { reported } \\
\text { experiencing } \\
\text { abuse regularly } \\
\text { were more } \\
\text { likely to be } \\
\text { HIV positive. }\end{array}$ & $\begin{array}{l}\text { Those who } \\
\text { reported } \\
\text { experiencing } \\
\text { abuse regularly } \\
\text { were more } \\
\text { likely to be a } \\
\text { current user } \\
\text { of sex-related } \\
\text { drugs. }\end{array}$ & $\begin{array}{l}\text { Those who } \\
\text { reported } \\
\text { experiencing } \\
\text { abuse regularly } \\
\text { were more } \\
\text { likely to have } \\
\text { exchanged } \\
\text { sex for } \\
\text { payment, } \\
\text { and be a } \\
\text { current user } \\
\text { of sex- } \\
\text { related } \\
\text { drugs. }\end{array}$ \\
\hline $\begin{array}{l}12) \text { Clum } \\
\text { (2009) [41] }\end{array}$ & $\begin{array}{l}40 \\
(40 / 0) .\end{array}$ & $\begin{array}{l}\text { USA. } 40 \\
\text { young HIV- } \\
\text { positive women } \\
\text { recruited } \\
\text { from HIV } \\
\text { clinics. }\end{array}$ & $\begin{array}{l}\text { Survey } \\
\text { HIV + only }\end{array}$ & $\begin{array}{l}\text { Mixed method } \\
\text { design } \\
\text { (qualitative } \\
\text { and quantitative } \\
\text { surveys). }\end{array}$ & $\begin{array}{l}\text { A modified version } \\
\text { of the Life Story } \\
\text { Interview was } \\
\text { used to cover } \\
\text { abuse experiences, } \\
\text { cognitive and } \\
\text { emotional } \\
\text { consequences of } \\
\text { abuse, coping } \\
\text { strategies, and } \\
\text { sexual behavior } \\
\text { and relationships. } \\
\text { PTSD symptoms } \\
\text { were assessed with } \\
\text { an interviewer- } \\
\text { administered } \\
\text { Posttraumatic } \\
\text { Diagnostic Scale. }\end{array}$ & $\begin{array}{l}\text { (1) PTSD } \\
\text { symptomatology } \\
\text { ranging from mild } \\
\text { to severe. } \\
\text { (2) Reported } \\
\text { difficulties in } \\
\text { sexual, family, } \\
\text { and friend } \\
\text { relationships, } \\
\text { general life } \\
\text { satisfaction, } \\
\text { and leisure time } \\
\text { activities. } \\
\text { (3) Substance } \\
\text { abuse. }\end{array}$ & $\begin{array}{l}75 \% \text { of the } \\
\text { women } \\
\text { reported } \\
\text { sexual abuse, } \\
80 \% \text { reported } \\
\text { physical abuse, } \\
\text { and } 55 \% \\
\text { reported } \\
\text { both types } \\
\text { of abuse. }\end{array}$ & $\begin{array}{l}\text { The average } \\
\text { PTSD score was } \\
20.75, \text { reflecting } \\
\text { moderate to } \\
\text { severe levels } \\
\text { of PTSD } \\
\text { symptoms. } \\
15 \% \text { of the } \\
\text { sample } \\
\text { reported mild } \\
\text { PTSD } \\
\text { symptoms } \\
\text { (<10), 37.5\% } \\
\text { reported } \\
\text { moderate } \\
\text { symptoms } \\
(10 \text { to 20), } \\
30 \% \text { reported } \\
\text { moderate to } \\
\text { severe symptoms } \\
(21 \text { to } 35), \text { and } \\
15 \% \text { reported } \\
\text { severe symptoms } \\
\text { ( }>35) \text {. Avoidance } \\
\text { and substance } \\
\text { use were } \\
\text { frequently } \\
\text { utilized as } \\
\text { coping strategies. }\end{array}$ & \\
\hline
\end{tabular}


Table $\mathbf{1}$ Summary of $\mathbf{3 4}$ articles selected for review (Continued)

\begin{tabular}{|c|c|c|c|c|c|c|c|c|c|c|}
\hline $\begin{array}{l}\text { (13) Cohen } \\
\text { (2004) [25] }\end{array}$ & $\begin{array}{l}1165 \\
(1165 / 0) .\end{array}$ & $\begin{array}{l}\text { USA. } 1165 \\
\text { HIV-positive } \\
\text { women. } 635 \\
\text { participants } \\
\text { were using } \\
\text { highly active } \\
\text { antiretroviral } \\
\text { therapy (HAART), } \\
254 \text { participants } \\
\text { not using } \\
\text { HAART although } \\
\text { it had been } \\
\text { indicated and } \\
\text { 276 participants } \\
\text { not on HAART } \\
\text { which had } \\
\text { not been } \\
\text { indicated. }\end{array}$ & $\begin{array}{l}\text { Survey } \\
\text { HIV + only }\end{array}$ & $\begin{array}{l}\text { Quantitative } \\
\text { survey } \\
\text { design. }\end{array}$ & $\begin{array}{l}\text { A standardized } \\
\text { interview-based } \\
\text { survey assessed } \\
\text { demographics, } \\
\text { medical and } \\
\text { psychosocial history, } \\
\text { history of } \\
\text { cigarette smoking, } \\
\text { alcohol use, illicit } \\
\text { drug use and drug } \\
\text { treatment programs, } \\
\text { sexual history and } \\
\text { history of medication } \\
\text { use, and reasons for } \\
\text { non-adherence at } \\
\text { each 6-month visit. } \\
\text { The CES-D } \\
\text { measured depressive } \\
\text { symptoms. Women } \\
\text { were also asked } \\
\text { questions about } \\
\text { physical, sexual, or } \\
\text { emotional coercion. }\end{array}$ & $\begin{array}{l}\text { (1) Poor } \\
\text { treatment } \\
\text { adherence. } \\
\text { (2). Drug abuse. } \\
\text { (3) High levels } \\
\text { of depression in } \\
\text { all groups. }\end{array}$ & $\begin{array}{l}72 \% \text { of women } \\
\text { using HAART } \\
\text { reported a } \\
\text { history of } \\
\text { physical or } \\
\text { sexual abuse. } \\
\text { For women } \\
\text { who were not } \\
\text { using HAART, } \\
80 \% \text { reported a } \\
\text { history of physical } \\
\text { or sexual abuse. } \\
\text { Current crack, } \\
\text { cocaine, or } \\
\text { heroin use, } \\
\text { being non-White, } \\
\text { and experiencing } \\
\text { any physical or } \\
\text { sexual abuse } \\
\text { increased the } \\
\text { likelihood of no } \\
\text { HAART use. }\end{array}$ & $\begin{array}{l}\text { A lower } \\
\text { percentage of } \\
\text { women with a } \\
\text { history of past } \\
\text { and current } \\
\text { use of crack, } \\
\text { cocaine, or } \\
\text { heroin were } \\
\text { using HAART. } \\
\text { Women in the } \\
\text { groups did } \\
\text { not differ } \\
\text { significantly } \\
\text { in having high } \\
\text { levels of } \\
\text { depressive } \\
\text { symptoms. }\end{array}$ & & $\begin{array}{l}\text { A lower } \\
\text { percentage of } \\
\text { women with } \\
\text { a history of } \\
\text { physical or } \\
\text { sexual abuse } \\
\text { reported } \\
\text { using HAART. } \\
\text { Women } \\
\text { who used } \\
\text { crack, cocaine, } \\
\text { or heroin in } \\
\text { the past year } \\
\text { were more } \\
\text { than twice } \\
\text { as likely to } \\
\text { report lack of } \\
\text { HAART use, } \\
\text { even when } \\
\text { indicated. } \\
\text { Similarly, } \\
\text { women with } \\
\text { a history of } \\
\text { any physical/ } \\
\text { sexual abuse } \\
\text { were more } \\
\text { than } 1.5 \text { times } \\
\text { more likely } \\
\text { to lack HAART } \\
\text { when clinically } \\
\text { eligible. }\end{array}$ \\
\hline $\begin{array}{l}\overline{(14)} \text { Gielen } \\
(2001)[26]\end{array}$ & $\begin{array}{l}287 \\
(287 / 0) .\end{array}$ & $\begin{array}{l}\text { USA. } 287 \\
\text { HIV-positive } \\
\text { women. 94\% } \\
\text { of the } \\
\text { women } \\
\text { were African- } \\
\text { American. }\end{array}$ & $\begin{array}{l}\text { Survey } \\
\text { HIV + only }\end{array}$ & $\begin{array}{l}\text { Quantitative } \\
\text { cross-sectional } \\
\text { survey } \\
\text { design. }\end{array}$ & $\begin{array}{l}\text { Health status and } \\
\text { quality of life were } \\
\text { evaluated with a } \\
\text { modified version of } \\
\text { the Medical Outcomes } \\
\text { Study HIV Health } \\
\text { Survey. HIV-related } \\
\text { characteristics, social } \\
\text { support and health } \\
\text { promoting behaviors } \\
\text { were assessed. Adult } \\
\text { violence history and } \\
\text { whether they had ever } \\
\text { been sexually abused } \\
\text { or raped as a child } \\
\text { was assessed using } \\
\text { a dichotomous } \\
\text { response variable } \\
\text { (yes/no). }\end{array}$ & $\begin{array}{l}\text { (1) Drug abuse. } \\
\text { (2) Poor mental } \\
\text { health, physical } \\
\text { functioning, } \\
\text { and quality } \\
\text { of life. } \\
\text { (3) Social } \\
\text { networks and } \\
\text { health } \\
\text { promoting } \\
\text { behaviors } \\
\text { improved } \\
\text { mental health. }\end{array}$ & $\begin{array}{l}\text { A history of CSA } \\
\text { was reported by } \\
41 \% \text { of the } \\
\text { sample. }\end{array}$ & $\begin{array}{l}55 \% \text { had a } \\
\text { history of } \\
\text { injection drug } \\
\text { use. Women } \\
\text { with a history } \\
\text { of child sexual } \\
\text { abuse reported } \\
\text { significantly } \\
\text { lower scores } \\
\text { on measures } \\
\text { of mental health, } \\
\text { physical } \\
\text { functioning, } \\
\text { and quality of } \\
\text { life. Women with } \\
\text { larger social } \\
\text { networks and } \\
\text { who practiced } \\
\text { more health } \\
\text { promoting } \\
\text { behaviors }\end{array}$ & $\begin{array}{l}63 \% \text { reported } \\
\text { having been } \\
\text { physically or } \\
\text { sexually } \\
\text { assaulted at } \\
\text { least once } \\
\text { as an adult. }\end{array}$ & \\
\hline
\end{tabular}




\begin{tabular}{|c|c|c|c|c|c|c|c|c|c|}
\hline & & & & & & & & $\begin{array}{l}\text { reported higher } \\
\text { levels of mental } \\
\text { health, whereas } \\
\text { those who had } \\
\text { been sexually } \\
\text { abused as } \\
\text { children } \\
\text { reported } \\
\text { significantly } \\
\text { worse mental } \\
\text { health. }\end{array}$ & \\
\hline $\begin{array}{l}\text { (15) Henny } \\
\text { (2007) [42] }\end{array}$ & $\begin{array}{l}644 \\
(644 / 0) .\end{array}$ & $\begin{array}{l}\text { USA. HIV- } \\
\text { seropositive } \\
\text { homeless or } \\
\text { unstably } \\
\text { housed adults } \\
\text { ( } n=644) \text {. } \\
\text { The sample } \\
\text { included } \\
15 \text { male-to- } \\
\text { female } \\
\text { transgender } \\
\text { people }\end{array}$ & $\begin{array}{l}\text { Survey } \\
\text { HIV + only }\end{array}$ & $\begin{array}{l}\text { Quantitative } \\
\text { cross-sectional } \\
\text { survey } \\
\text { design. }\end{array}$ & $\begin{array}{l}\text { Dichotomous } \\
\text { variables } \\
\text { (yes/no) assessed } \\
\text { adult and childhood } \\
\text { abuse, and current } \\
\text { and lifetime illicit } \\
\text { drug use. The CAGE } \\
\text { questionnaire } \\
\text { investigated alcohol } \\
\text { use. Depressive } \\
\text { symptoms were } \\
\text { measured by the } \\
\text { CES-D. Self-perception } \\
\text { of stress was } \\
\text { measured using the } \\
\text { Perceived Stress Scale. }\end{array}$ & $\begin{array}{l}\text { (1) Alcohol } \\
\text { abuse. } \\
\text { (2) Depressive } \\
\text { symptomatology. } \\
\text { (3) Transactional } \\
\text { sex. }\end{array}$ & $\begin{array}{l}80.3 \% \text { of the } \\
\text { sample reported } \\
\text { a history of any } \\
\text { physical or } \\
\text { sexual abuse. } \\
53 \% \text { reported } \\
\text { childhood } \\
\text { physical abuse } \\
\text { and } 38.7 \% \\
\text { reported } \\
\text { CSA. Victims } \\
\text { of CSA were } \\
\text { nearly three } \\
\text { times as likely } \\
\text { to be female. }\end{array}$ & $\begin{array}{l}\text { Victims of } \\
\text { childhood } \\
\text { physical abuse } \\
\text { were more } \\
\text { likely to have } \\
\text { abused alcohol. } \\
\text { Persons } \\
\text { experiencing } \\
\text { childhood } \\
\text { physical abuse } \\
\text { also were twice } \\
\text { as likely to } \\
\text { report } \\
\text { symptoms } \\
\text { indicating } \\
\text { depression. }\end{array}$ & $\begin{array}{l}\text { Persons } \\
\text { experiencing } \\
\text { childhood } \\
\text { physical abuse } \\
\text { also were } \\
\text { twice as } \\
\text { likely to have } \\
\text { ever exchanged } \\
\text { sex for money, } \\
\text { drugs, or } \\
\text { shelter. }\end{array}$ \\
\hline $\begin{array}{l}\text { (16) Kalichman } \\
\text { (2002) [28] }\end{array}$ & $\begin{array}{l}357 \\
(357 / 0) .\end{array}$ & $\begin{array}{l}\text { USA. } 357 \text { men } \\
\text { and women } \\
\text { living with } \\
\text { HIV/AIDS. Study } \\
\text { participants } \\
\text { were } 242 \\
(68 \%) \text { men, } \\
110(31 \%) \\
\text { women, and } \\
5(1 \%) \\
\text { transgender } \\
\text { persons. } \\
\text { The majority } \\
\text { of the sample } \\
\text { was African- } \\
\text { American } \\
\text { (76\%). }\end{array}$ & $\begin{array}{l}\text { Survey } \\
\text { HIV + only }\end{array}$ & $\begin{array}{l}\text { Quantitative } \\
\text { cross-sectional } \\
\text { survey } \\
\text { design. }\end{array}$ & $\begin{array}{l}\text { A dichotomous } \\
\text { variable (yes/no) } \\
\text { assessed sexual } \\
\text { abuse history and } \\
\text { substance abuse } \\
\text { history. Trauma } \\
\text { indicators were } \\
\text { adapted from } \\
\text { diagnostic symptoms } \\
\text { of PTSD. Symptoms } \\
\text { of depression were } \\
\text { assessed with the } \\
\text { CES-D. The Trait- } \\
\text { Anxiety Scale } \\
\text { assessed anxiety. } \\
\text { A 6-item } \\
\text { scale to assess } \\
\text { pessimism was } \\
\text { developed. } \\
\text { Symptoms } \\
\text { of obsessiveness- } \\
\text { compulsiveness } \\
\text { were assessed } \\
\text { using six items }\end{array}$ & $\begin{array}{l}\text { (1) Substance } \\
\text { abuse. } \\
\text { (2) Anxiety } \\
\text { symptoms. } \\
\text { (3) Depression } \\
\text { symptoms.(4) } \\
\text { Borderline } \\
\text { personality } \\
\text { symptoms.(5) } \\
\text { Current PTSD } \\
\text { symptoms. } \\
\text { (6) Trauma } \\
\text { symptoms } \\
\text { correlated with } \\
\text { the number of } \\
\text { sexual assaults } \\
\text { reported. }\end{array}$ & $\begin{array}{l}68 \% \text { of women } \\
\text { and } 35 \% \text { of men } \\
\text { living with } \\
\text { HIV/AIDS } \\
\text { reported a } \\
\text { history of } \\
\text { sexual assault } \\
\text { since age } 15 .\end{array}$ & $\begin{array}{l}\text { History of } \\
\text { sexual assault } \\
\text { was related to } \\
\text { history of } \\
\text { substance use } \\
\text { and mental } \\
\text { health treatment. } \\
\text { Sexual assault } \\
\text { survivors } \\
\text { reported greater } \\
\text { anxiety, depression, } \\
\text { and symptoms } \\
\text { of borderline } \\
\text { personality than } \\
\text { persons who had } \\
\text { not been sexually } \\
\text { assaulted. Persons } \\
\text { who reported } \\
\text { having been } \\
\text { sexually assaulted } \\
\text { reported current } \\
\text { trauma symptoms. } \\
\text { Specifically, } 24 \% \\
\text { stated that they }\end{array}$ & \\
\hline
\end{tabular}


Table 1 Summary of $\mathbf{3 4}$ articles selected for review (Continued)

\begin{tabular}{|c|c|c|c|c|c|c|c|c|c|}
\hline & & & & & $\begin{array}{l}\text { from the Obsessive- } \\
\text { Compulsive Scale } \\
\text { of the schedule } \\
\text { for nonadaptive } \\
\text { personality (SNAP). } \\
\text { Similarly, } \\
\text { six items from the } \\
\text { Borderline } \\
\text { Personality } \\
\text { Scale of the } \\
\text { SNAP were } \\
\text { used to assess } \\
\text { borderline } \\
\text { personality } \\
\text { characteristics. }\end{array}$ & & & $\begin{array}{l}\text { think of the } \\
\text { experience on a } \\
\text { regular basis, } 20 \% \\
\text { have nightmares } \\
\text { about the } \\
\text { experience, } 60 \% \\
\text { reported that } \\
\text { the experience } \\
\text { affects them today, } \\
\text { and } 47 \% \text { stated } \\
\text { that the experience } \\
\text { interferes with } \\
\text { their relationships. } \\
\text { Number of trauma } \\
\text { symptoms } \\
\text { correlated with } \\
\text { the number of } \\
\text { sexual assault } \\
\text { experiences } \\
\text { reported. }\end{array}$ & \\
\hline $\begin{array}{l}17) \text { Kang } \\
\text { (2008) [43] }\end{array}$ & $\begin{array}{l}220 \\
(220 / 0) .\end{array}$ & $\begin{array}{l}\text { USA. All } \\
\text { participants } \\
\text { were HIV- } \\
\text { positive } \\
\text { heroin and/or } \\
\text { crack cocaine } \\
\text { using African- } \\
\text { Americans } \\
\text { or Hispanics. } \\
\text { There were } \\
146 \text { males } \\
\text { and } 74 \\
\text { females. }\end{array}$ & $\begin{array}{l}\text { Survey } \\
\text { HIV + only }\end{array}$ & $\begin{array}{l}\text { Baseline } \\
\text { survey for } \\
\text { an intervention } \\
\text { study. }\end{array}$ & $\begin{array}{l}\text { Childhood abuse } \\
\text { experience: CTQ. } \\
\text { Depression: } \\
\text { CES-D. Health } \\
\text { status items } \\
\text { included: general } \\
\text { health rating } \\
\text { and HIV-related } \\
\text { symptoms. } \\
\text { Lifetime medical } \\
\text { conditions } \\
\text { were also } \\
\text { examined. }\end{array}$ & $\begin{array}{l}\text { (1) Alcohol and } \\
\text { drug abuse. } \\
\text { (2) High } \\
\text { depression } \\
\text { levels. } \\
\text { (3) Poor } \\
\text { treatment } \\
\text { adherence. }\end{array}$ & $\begin{array}{l}\text { Women were } \\
\text { more likely to } \\
\text { report CSA } \\
(51 \% \text { versus } \\
39 \%) \text { and } \\
\text { childhood } \\
\text { physical abuse } \\
(64 \% \text { versus } \\
54 \%) .\end{array}$ & $\begin{array}{l}\text { Men were more } \\
\text { likely to use } \\
\text { alcohol to } \\
\text { intoxication } \\
\text { and currently } \\
\text { inject drugs, } \\
\text { and females } \\
\text { were more } \\
\text { likely to use } \\
\text { crack. Both men } \\
\text { and women } \\
\text { had high } \\
\text { depression } \\
\text { levels. } 81 \% \text { of } \\
\text { women and } \\
76 \% \text { of men } \\
\text { had a score } \\
\text { of } 16 \text { or higher } \\
\text { on the } \\
\text { CES-D. }\end{array}$ & $\begin{array}{l}\text { For both } \\
\text { men and } \\
\text { women, } \\
\text { use of HIV } \\
\text { medications } \\
\text { was negatively } \\
\text { associated } \\
\text { with CSA } \\
\text { experience. }\end{array}$ \\
\hline $\begin{array}{l}\text { (18) Kimerling } \\
\left(1999^{\mathrm{a}}\right)[44]\end{array}$ & $\begin{array}{l}67 \\
(67 / 0) .\end{array}$ & $\begin{array}{l}\text { USA. Sample } \\
\text { included } \\
67 \text { African- } \\
\text { American } \\
\text { HIV-infected } \\
\text { women beyond } \\
\text { the initial } \\
\text { stages of } \\
\text { HIV infection. }\end{array}$ & $\begin{array}{l}\text { Survey } \\
\text { HIV + only }\end{array}$ & $\begin{array}{l}\text { Longitudinal } \\
\text { design: } \\
\text { 12-14 months } \\
\text { apart, with } \\
\text { an average time } \\
\text { of } 13.4 \text { months } \\
\text { apart. }\end{array}$ & $\begin{array}{l}\text { Life Stressor } \\
\text { Checklist: } \\
\text { identify life } \\
\text { stressors } \\
\text { with greater } \\
\text { prevalence } \\
\text { for women. } \\
\text { Impact of } \\
\text { Events }\end{array}$ & $\begin{array}{l}\text { (1) PTSD (both } \\
\text { symptom clusters } \\
\text { and full criteria). }\end{array}$ & $\begin{array}{l}62 \% \text { of the } \\
\text { sample } \\
\text { reported } \\
\text { experiencing } \\
\text { at least one } \\
\text { traumatic event. } \\
30 \% \text { of the } \\
\text { sample } \\
\text { experienced }\end{array}$ & $\begin{array}{l}\text { The majority } \\
\text { who met the } \\
\text { stressor criterion } \\
\text { also met criteria } \\
\text { for at least one } \\
\text { other symptom } \\
\text { cluster for } \\
\text { PTSD, whereas } \\
35 \% \text { of the }\end{array}$ & \\
\hline
\end{tabular}


Table 1 Summary of $\mathbf{3 4}$ articles selected for review (Continued)

\begin{tabular}{|c|c|c|c|c|c|c|c|c|}
\hline & & & & & $\begin{array}{l}\text { Scale-Revised: } \\
\text { the presence } \\
\text { and intensity } \\
\text { of PTSD } \\
\text { symptoms. }\end{array}$ & & $\begin{array}{l}\text { completed rape } \\
\text { and } 33 \% \\
\text { experienced } \\
\text { physical assault. } \\
\text { These both } \\
\text { included rape } \\
\text { and assault as } \\
\text { a child. }\end{array}$ & $\begin{array}{l}\text { sample met } \\
\text { full criteria. } \\
88 \% \text { of the } \\
\text { sample met } \\
\text { criteria for the } \\
\text { re-experiencing } \\
\text { symptom cluster, } \\
74 \% \text { for the } \\
\text { avoidance/ } \\
\text { numbing } \\
\text { symptom } \\
\text { cluster and } \\
70 \% \text { for the } \\
\text { hyper arousal } \\
\text { symptom cluster. }\end{array}$ \\
\hline $\begin{array}{l}\text { (19) Martinez } \\
\text { (2002) [45] }\end{array}$ & $\begin{array}{l}41 \\
(41 / 0) .\end{array}$ & $\begin{array}{l}\text { USA. } 41 \\
\text { HIV-positive } \\
\text { women. The } \\
\text { majority } \\
\text { of the sample } \\
\text { (51\%) was } \\
\text { African- } \\
\text { American. }\end{array}$ & $\begin{array}{l}\text { Survey } \\
\text { HIV + only }\end{array}$ & $\begin{array}{l}\text { Quantitative } \\
\text { cross-sectional } \\
\text { survey design: } \\
\text { retrospective } \\
\text { data. }\end{array}$ & $\begin{array}{l}\text { The Life Stressor } \\
\text { Checklist-Revised } \\
\text { was completed in } \\
\text { order to examine } \\
\text { the frequency and } \\
\text { types of traumatic } \\
\text { life events. The } \\
\text { PTSD Checklist- } \\
\text { Civilian Version } \\
29 \text { was used } \\
\text { to assess current } \\
\text { PTSD symptoms. }\end{array}$ & $\begin{array}{l}\text { (1) Partial and } \\
\text { full PTSD. } \\
\text { (2) Level of } \\
\text { PTSD } \\
\text { significantly } \\
\text { related to } \\
\text { number of } \\
\text { life events } \\
\text { experienced } \\
\text { and perceived } \\
\text { social support. }\end{array}$ & $\begin{array}{l}61 \% \text { of women } \\
\text { had experienced } \\
\text { growing up } \\
\text { with violence } \\
\text { in the home. } \\
59 \% \text { were } \\
\text { emotionally } \\
\text { abused or } \\
\text { neglected. } 32 \% \\
\text { had been abused } \\
\text { or physically } \\
\text { attacked by a } \\
\text { known person } \\
\text { before the age } \\
\text { of } 16 . \text { Similarly, } \\
32 \% \text { were } \\
\text { sexually } \\
\text { touched or } \\
\text { made to touch } \\
\text { someone before } \\
\text { age } 16 \text { and } 31 \% \\
\text { were forced to } \\
\text { have some type } \\
\text { of sex before } \\
\text { age } 16 \text {. }\end{array}$ & $\begin{array}{l}42 \% \text { of the } \\
\text { HIV-positive } \\
\text { women were } \\
\text { likely to meet } \\
\text { criteria for full } \\
\text { current PTSD } \\
\text { and an } \\
\text { additional 22\% } \\
\text { for partial PTSD. } \\
\text { Women reported } \\
\text { having } \\
\text { experienced a } \\
\text { mean of } 12 \\
\text { traumatic life } \\
\text { events. The level } \\
\text { of PTSD was } \\
\text { significantly } \\
\text { related to the } \\
\text { number of life } \\
\text { events } \\
\text { experienced } \\
\text { and to } \\
\text { perceived } \\
\text { social support } \\
\text { from friends } \\
\text { and family. }\end{array}$ \\
\hline $\begin{array}{l}\text { (20) Martinez } \\
\text { (2009) [46] }\end{array}$ & $\begin{array}{l}174 \\
(174 / 0) .\end{array}$ & $\begin{array}{l}\text { USA. HIV- } \\
\text { positive youth } \\
\text { enrolled in } \\
\text { a young } \\
\text { adult HIV } \\
\text { clinic between } \\
1998 \text { and } \\
2006.58 \text { were } \\
\text { females }\end{array}$ & $\begin{array}{l}\text { Survey } \\
\text { HIV + only }\end{array}$ & $\begin{array}{l}\text { Quantitative } \\
\text { cross-sectional } \\
\text { survey } \\
\text { design. }\end{array}$ & $\begin{array}{l}\text { Client Diagnostic } \\
\text { Questionnaire } \\
\text { was used to } \\
\text { screen for } \\
\text { mental health } \\
\text { disorders and } \\
\text { violence. } \\
\text { All youth } \\
\text { subsequently had }\end{array}$ & $\begin{array}{l}\text { (1) Major } \\
\text { depressive } \\
\text { disorder (MDD). } \\
\text { (2) Generalized } \\
\text { anxiety disorder. } \\
\text { (3) PTSD. } \\
\text { (4) Alcohol and } \\
\text { substance } \\
\text { abuse disorders. }\end{array}$ & $\begin{array}{l}\text { Violence reported } \\
\text { included physical } \\
\text { abuse (24\% in } \\
\text { childhood; } 19 \% \\
\text { in adolescents), } \\
\text { sexual abuse } \\
\text { (28\% in } \\
\text { childhood; } 15 \% \\
\text { in adolescents), }\end{array}$ & $\begin{array}{l}\text { Psychological } \\
\text { disorders included: } \\
\text { MDD (15\%), } \\
\text { generalized anxiety } \\
\text { disorder (17\%); } \\
\text { PTSD (28\%); } \\
\text { alcohol abuse } \\
\text { disorder (19\%); } \\
\text { and substance }\end{array}$ \\
\hline
\end{tabular}


Table 1 Summary of $\mathbf{3 4}$ articles selected for review (Continued)

\begin{tabular}{|c|c|c|c|c|c|c|c|c|c|}
\hline & & $\begin{array}{l}\text { and } 116 \text { were } \\
\text { males. } \\
\text { The majority } \\
\text { (79\%) } \\
\text { were African- } \\
\text { American. }\end{array}$ & & & $\begin{array}{l}\text { diagnostic } \\
\text { interviews } \\
\text { conducted by } \\
\text { psychologists. }\end{array}$ & & $\begin{array}{l}\text { dating violence } \\
(18 \%) \text {, and family } \\
\text { violence }(44 \%) \text {. } \\
\text { Females had } \\
\text { higher sexual } \\
\text { abuse }(P<.001) \text {. }\end{array}$ & $\begin{array}{l}\text { abuse disorder } \\
\text { ( } 31 \%) \text {. Physically } \\
\text { abused youth } \\
\text { had higher } \\
\text { symptoms of } \\
\text { anxiety and } \\
\text { PTSD. Sexually } \\
\text { abused youth } \\
\text { had higher } \\
\text { symptoms of } \\
\text { PTSD ( } P<0.05) \text {. } \\
\text { Youth with } \\
\text { family violence } \\
\text { had higher } \\
\text { symptoms of } \\
\text { Anxiety Disorder } \\
(P<0.05) \text { and } \\
\text { PTSD }(P<0.01) \text {. }\end{array}$ & \\
\hline $\begin{array}{l}\text { (21) McKeown } \\
\text { (2003) [47] }\end{array}$ & $\begin{array}{l}20 \\
(20 / 0) .\end{array}$ & $\begin{array}{l}\text { Canada. } \\
20 \text { HIV-positive } \\
\text { women. } \\
\text { Eighteen } \\
(90 \%) \\
\text { self-identified } \\
\text { as aboriginal. }\end{array}$ & $\begin{array}{l}\text { Survey } \\
\text { HIV + only }\end{array}$ & $\begin{array}{l}\text { Qualitative } \\
\text { research } \\
\text { design. }\end{array}$ & $\begin{array}{l}\text { Open ended } \\
\text { interviews were } \\
\text { conducted } \\
\text { to obtain } \\
\text { information on } \\
\text { childhood and } \\
\text { adulthood } \\
\text { experiences. }\end{array}$ & $\begin{array}{l}\text { (1) Drug abuse } \\
\text { as coping } \\
\text { strategy. } \\
\text { (2) Transactional } \\
\text { sex. } \\
\text { (3) Past suicide } \\
\text { attempts. } \\
\text { (4) Reported } \\
\text { diagnoses of } \\
\text { MDD, PTSD, } \\
\text { schizophrenia, } \\
\text { panic disorder, } \\
\text { and multiple } \\
\text { personality } \\
\text { disorder. }\end{array}$ & $\begin{array}{l}\text { Women who } \\
\text { had experienced } \\
\text { CSA. }\end{array}$ & $\begin{array}{l}\text { A few of the } \\
\text { women } \\
\text { recounted past } \\
\text { attempts of } \\
\text { suicide. A } \\
\text { number of } \\
\text { women, at the } \\
\text { time of the } \\
\text { interview, } \\
\text { reported a } \\
\text { diagnosis of } \\
\text { mental illness } \\
\text { including } \\
\text { depression, } \\
\text { multiple } \\
\text { personality } \\
\text { disorder, panic } \\
\text { attacks, PTSD } \\
\text { and } \\
\text { schizophrenia. } \\
\text { Most participants } \\
\text { reported IVDU } \\
\text { on a regular basis } \\
\text { in the past, with } \\
\text { one reporting } \\
\text { current use of } \\
\text { IV drugs. }\end{array}$ & $\begin{array}{l}\text { The majority } \\
\text { who experienced } \\
\text { CSA reported } \\
\text { involvement } \\
\text { in the sex } \\
\text { trade and } \\
\text { drug abuse } \\
\text { as economic } \\
\text { and emotional } \\
\text { survival/ } \\
\text { coping } \\
\text { strategies. }\end{array}$ \\
\hline $\begin{array}{l}\text { (22) Meade } \\
\text { (2009) [7] }\end{array}$ & $\begin{array}{l}271 \\
(271 / 0) .\end{array}$ & $\begin{array}{l}\text { USA. } 271 \\
\text { HIV-positive } \\
\text { individuals } \\
\text { with histories } \\
\text { of CSA. 50\% }\end{array}$ & $\begin{array}{l}\text { Survey } \\
\text { HIV + only }\end{array}$ & $\begin{array}{l}\text { Baseline } \\
\text { survey } \\
\text { for a coping } \\
\text { intervention } \\
\text { trial }\end{array}$ & $\begin{array}{l}\text { A modified version } \\
\text { of the TEQ was used } \\
\text { to verify childhood } \\
\text { abuse history. } \\
\text { The BDI was used }\end{array}$ & $\begin{array}{l}\text { (1) Depressive } \\
\text { disorder. } \\
\text { (2) Anxiety } \\
\text { disorder. } \\
\text { (3) Psychotic }\end{array}$ & $\begin{array}{l}271 \text { HIV-positive } \\
\text { individuals with } \\
\text { histories of CSA. }\end{array}$ & $\begin{array}{l}\text { Approximately } \\
\text { half of the } \\
\text { sample (53\%) } \\
\text { screened } \\
\text { positive for }\end{array}$ & \\
\hline
\end{tabular}


Table 1 Summary of $\mathbf{3 4}$ articles selected for review (Continued)

\begin{tabular}{|c|c|c|c|c|c|c|c|c|}
\hline & & $\begin{array}{l}\text { female and } \\
69 \% \text { African- } \\
\text { American. } \\
\text { The men } \\
\text { were primarily } \\
\text { (94\%) gay/ } \\
\text { bisexual. }\end{array}$ & & & $\begin{array}{l}\text { to identify severe } \\
\text { depression. }\end{array}$ & $\begin{array}{l}\text { disorder. } \\
\text { (4) Adjustment } \\
\text { disorder. } \\
\text { (5) Bipolar } \\
\text { disorder. } \\
\text { (6) Alcohol and } \\
\text { drug abuse. } \\
\text { (7) Undergone } \\
\text { mental health } \\
\text { treatment. }\end{array}$ & & $\begin{array}{l}\text { one or more } \\
\text { psychiatric } \\
\text { disorders } \\
\text { (30\% depressive, } \\
25 \% \text { anxiety, } \\
11 \% \text { psychotic, } \\
10 \% \text { adjustment, } \\
4 \% \text { bipolar). } \\
\text { Approximately } \\
\text { one third (37\%) } \\
\text { used illicit drugs } \\
\text { and } 10 \% \text { reported } \\
\text { binge drinking in } \\
\text { the past } 4 \text { months. } \\
\text { Many participants } \\
\text { also received } \\
\text { mental health } \\
\text { treatment in the } \\
\text { past } 4 \text { months. } \\
\text { Those screening } \\
\text { positive for a } \\
\text { psychiatric } \\
\text { disorder were } \\
\text { more likely than } \\
\text { those who did } \\
\text { not to have } \\
\text { received mental } \\
\text { health treatment } \\
\text { (59\% versus } \\
41 \% \text { ). }\end{array}$ \\
\hline $\begin{array}{l}\text { (23) Pence } \\
\text { (2007) [29] }\end{array}$ & $\begin{array}{l}611 \\
(611 / 0) .\end{array}$ & $\begin{array}{l}\text { USA. } 611 \\
\text { HIV-infected } \\
\text { individuals. } \\
\text { Sixty four } \\
\text { percent of } \\
\text { participants } \\
\text { were African- } \\
\text { American } \\
\text { and } 31 \% \\
\text { were female. }\end{array}$ & $\begin{array}{l}\text { Survey } \\
\text { HIV + only }\end{array}$ & $\begin{array}{l}\text { Quantitative } \\
\text { cross-sectional } \\
\text { survey } \\
\text { design. }\end{array}$ & $\begin{array}{l}\text { Patients completed } \\
\text { the Brief Symptoms } \\
\text { Inventory (BSI), an } \\
\text { assessment } \\
\text { of current } \\
\text { psychological } \\
\text { symptoms. } \\
\text { Substance } \\
\text { use was measured } \\
\text { with the Addiction } \\
\text { Severity Index. } \\
\text { PTSD symptoms } \\
\text { were assessed } \\
\text { with the } \\
\text { PTSD Checklist. }\end{array}$ & $\begin{array}{l}\text { (1) PTSD. } \\
\text { (2) More than } \\
\text { half had a } \\
\text { probable } \\
\text { psychiatric } \\
\text { disorder on } \\
\text { the BSI. } \\
\text { (3) High levels } \\
\text { of depression. } \\
\text { (4) High levels } \\
\text { of anxiety. } \\
\text { (5) Substance } \\
\text { abuse. }\end{array}$ & $\begin{array}{l}\text { Most respondents } \\
(91 \%) \text { reported } \\
\text { experiencing at } \\
\text { least one traumatic } \\
\text { event in their } \\
\text { lifetime. } \\
30.4 \% \text { experienced } \\
\text { CSA and } 20.6 \% \\
\text { severe physical } \\
\text { abuse as a child. }\end{array}$ & $\begin{array}{l}16 \% \text { of the sample } \\
\text { met criteria for } \\
\text { PTSD, } 53.9 \% \text { of } \\
\text { the sample had } \\
\text { a probable } \\
\text { psychiatric } \\
\text { disorder on } \\
\text { the BSI. } 34.7 \% \\
\text { of the sample } \\
\text { had depressive } \\
\text { symptoms above } \\
\text { the 90th } \\
\text { percentile } \\
\text { and } 29.5 \% \\
\text { had anxiety } \\
\text { symptoms above }\end{array}$ \\
\hline
\end{tabular}




\begin{tabular}{|c|c|c|c|c|c|c|c|c|c|}
\hline & & & & & & & & $\begin{array}{l}\text { the 90th } \\
\text { percentile. } \\
22.3 \% \text { of the } \\
\text { sample was } \\
\text { engaging in } \\
\text { any non- } \\
\text { marijuana } \\
\text { substance } \\
\text { abuse and 20\% } \\
\text { were using } \\
\text { multiple } \\
\text { substances. }\end{array}$ & \\
\hline $\begin{array}{l}\text { (24) Sikkema } \\
\text { (2009) [48] }\end{array}$ & $\begin{array}{l}256 \\
(256 / 0) .\end{array}$ & $\begin{array}{l}\text { USA. } 256 \\
\text { HIV-positive } \\
\text { adults with } \\
\text { CSA histories. } \\
\text { There were } \\
132 \text { women } \\
\text { and } 124 \text { MSM. } \\
\text { The majority } \\
\text { (67.3\%) was } \\
\text { African- } \\
\text { American. }\end{array}$ & $\begin{array}{l}\text { Survey } \\
\text { HIV + only }\end{array}$ & $\begin{array}{l}\text { Quantitative } \\
\text { cross-sectional } \\
\text { survey } \\
\text { design. }\end{array}$ & $\begin{array}{l}\text { A modified version } \\
\text { of the TEQ assessed } \\
\text { abuse history. } \\
\text { Depression and } \\
\text { suicidal ideation: } \\
\text { BDI. TSI: PTSD. } \\
\text { Substance abuse } \\
\text { and sexual behavior } \\
\text { were also assessed } \\
\text { using self- } \\
\text { developed } \\
\text { screening tools. }\end{array}$ & $\begin{array}{l}\text { (1) Sexual } \\
\text { revictimization. } \\
\text { (2) Mood and } \\
\text { anxiety } \\
\text { symptoms. } \\
\text { (3) PTSD } \\
\text { symptoms. } \\
\text { (4) Alcohol and } \\
\text { drug use. }\end{array}$ & $\begin{array}{l}\text { All participants } \\
\text { reported abuse } \\
\text { histories. } \\
90 \% \text { had } \\
\text { experienced } \\
\text { penetrative } \\
\text { vaginal } \\
\text { or anal sexual } \\
\text { abuse as a child } \\
\text { or adolescent. }\end{array}$ & $\begin{array}{l}\text { The mean score } \\
\text { for mood and } \\
\text { anxiety } \\
\text { symptoms } \\
\text { was } 29.8 \text { in } \\
\text { women and } \\
28.2 \text { in men. } \\
\text { Mean score for } \\
\text { trauma-related } \\
\text { symptoms was } \\
40.4 \text { in women } \\
\text { and } 28.9 \text { in men. } \\
\text { Alcohol use in } \\
\text { the past } \\
4 \text { months } \\
\text { was } 31.8 \% \text { in } \\
\text { women and } \\
53.2 \% \text { in men. } \\
\text { Marijuana use } \\
\text { in the past } \\
4 \text { months was } \\
18.2 \% \text { in women } \\
\text { and } 36.3 \% \text { in } \\
\text { men. Cocaine } \\
\text { and/or Crack use } \\
\text { in the past } \\
4 \text { months } \\
\text { was } 18.9 \% \text { in } \\
\text { women and } \\
33.1 \% \text { in men. }\end{array}$ & $\begin{array}{l}87 \% \text { experienced } \\
\text { sexual } \\
\text { revictimization } \\
\text { at some point } \\
\text { in their lives. }\end{array}$ \\
\hline $\begin{array}{l}\text { (25) Simoni } \\
\text { (2000) [49] }\end{array}$ & $\begin{array}{l}230 \\
(230 / 0) .\end{array}$ & $\begin{array}{l}\text { USA. Sample } \\
\text { consisted } \\
\text { of } 230 \\
\text { HIV-positive } \\
\text { women. } \\
\text { The majority } \\
(46 \%) \\
\text { described }\end{array}$ & $\begin{array}{l}\text { Survey } \\
\text { HIV + only }\end{array}$ & $\begin{array}{l}\text { Quantitative } \\
\text { cross-sectional } \\
\text { survey } \\
\text { design. }\end{array}$ & $\begin{array}{l}\text { Demographics, } \\
\text { trauma, coping } \\
\text { strategies and } \\
\text { current } \\
\text { depressive } \\
\text { symptomatology } \\
\text { were assessed. } \\
\text { Respondents }\end{array}$ & $\begin{array}{l}\text { (1) High scores } \\
\text { of depressive } \\
\text { symptoms. } \\
\text { (2) Positive } \\
\text { correlation } \\
\text { between } \\
\text { childhood abuse } \\
\text { and current }\end{array}$ & $\begin{array}{l}\text { A high prevalence } \\
\text { of abuse in } \\
\text { childhood (50\%) } \\
\text { and adulthood } \\
(68 \%) ; 7 \% \\
\text { reported physical } \\
\text { assault or rape }\end{array}$ & $\begin{array}{l}\text { Childhood abuse } \\
\text { was significantly } \\
\text { correlated with } \\
\text { both adult and } \\
\text { recent trauma, } \\
\text { and each type of } \\
\text { trauma correlated } \\
\text { with CES-D scores. }\end{array}$ & \\
\hline
\end{tabular}


Table 1 Summary of $\mathbf{3 4}$ articles selected for review (Continued)

\begin{tabular}{|c|c|c|c|c|c|c|c|c|c|}
\hline & & $\begin{array}{l}\text { themselves } \\
\text { as African- } \\
\text { American. }\end{array}$ & & & $\begin{array}{l}\text { completed the } \\
\text { CES-D. Self- } \\
\text { reported trauma } \\
\text { histories were } \\
\text { documented. }\end{array}$ & $\begin{array}{l}\text { adaptive and } \\
\text { avoidant coping } \\
\text { strategies. } \\
\text { (3) Avoidant } \\
\text { coping was } \\
\text { strongly } \\
\text { associated with } \\
\text { CES-D scores. }\end{array}$ & $\begin{array}{l}\text { in the last } \\
90 \text { days. }\end{array}$ & $\begin{array}{l}\text { The mean CES-D } \\
\text { score was } 22.49 ; \\
66 \% \text { had a sum } \\
\text { score of } 16 \text { or } \\
\text { above, indicative } \\
\text { of possible } \\
\text { clinical } \\
\text { depression. }\end{array}$ & \\
\hline $\begin{array}{l}\text { (26) Tarakeshwar } \\
\text { (2005) [50] }\end{array}$ & $\begin{array}{l}28 \\
(28 / 0)\end{array}$ & $\begin{array}{l}\text { USA. } 28 \\
\text { HIV-positive } \\
\text { women with } \\
\text { CSA histories. } \\
\text { The majority } \\
\text { were African- } \\
\text { American } \\
\text { (67.9\%). }\end{array}$ & $\begin{array}{l}\text { Survey } \\
\text { HIV + only }\end{array}$ & $\begin{array}{l}\text { Qualitative } \\
\text { research } \\
\text { design. }\end{array}$ & $\begin{array}{l}\text { A clinical psychologist } \\
\text { and a social worker } \\
\text { conducted in-depth } \\
\text { qualitative interviews. } \\
\text { The interview was } \\
\text { developed on the } \\
\text { basis of the } \\
\text { published } \\
\text { literature and the } \\
\text { goal of developing } \\
\text { a coping- } \\
\text { focused intervention } \\
\text { for women with } \\
\text { CSA history } \\
\text { and HIV. The } \\
\text { interview protocol } \\
\text { used a semi- } \\
\text { structured } \\
\text { interview format } \\
\text { that addressed } \\
\text { the impact } \\
\text { of sexual abuse } \\
\text { and HIV on } \\
\text { their life and } \\
\text { the ways they } \\
\text { coped with these } \\
\text { traumas. }\end{array}$ & $\begin{array}{l}\text { (1) Reported } \\
\text { cumulative } \\
\text { trauma-related } \\
\text { distress. } \\
\text { (2) Current use } \\
\text { of psychiatric } \\
\text { medications for: } \\
\text { depression, } \\
\text { anxiety } \\
\text { (agoraphobia, } \\
\text { panic disorder), } \\
\text { PTSD. } \\
\text { (3) Frequent } \\
\text { hospital visits } \\
\text { for physical } \\
\text { complaints. } \\
\text { (4) Substance } \\
\text { abuse. } \\
\text { (5) Revictimization. }\end{array}$ & $\begin{array}{l}78.6 \% \text { of the } \\
\text { sample revealed } \\
\text { unwanted touching } \\
\text { or fondling, } \\
57.1 \% \text { reported } \\
\text { sexual intercourse, } \\
\text { and } 57.1 \% \text { were } \\
\text { asked to engage } \\
\text { in sexual acts under } \\
\text { verbal and } \\
\text { emotional pressure } \\
\text { (before 13). } \\
\text { During adolescence } \\
\text { (13-17 years), } \\
\text { their reports of } \\
\text { unwanted sexual } \\
\text { abuse experiences } \\
\text { increased: } 82.1 \% \\
\text { for intercourse, } \\
64.3 \% \text { for oral sex, } \\
71.4 \% \text { for forced or } \\
\text { threatened sexual } \\
\text { acts, } 75.0 \% \text { for } \\
\text { verbal and } \\
\text { emotional } \\
\text { pressure, and } \\
35.7 \% \text { for } \\
\text { unwanted } \\
\text { sexual acts that } \\
\text { occurred when } \\
\text { they had passed } \\
\text { out or were drunk } \\
\text { or asleep. Many } \\
\text { (40\%) of the } \\
\text { women were } \\
\text { abused by family } \\
\text { members. }\end{array}$ & $\begin{array}{l}\text { Most of the } \\
\text { women } \\
\text { reported having } \\
\text { encountered } \\
\text { multiple } \\
\text { traumatic } \\
\text { experiences and } \\
\text { reported } \\
\text { cumulative } \\
\text { distress as a } \\
\text { result of these } \\
\text { experiences. } \\
\text { Many were } \\
\text { using psychiatric } \\
\text { medications for } \\
\text { symptoms of } \\
\text { depression, anxiety } \\
\text { (e.g., agoraphobia, } \\
\text { panic disorder), } \\
\text { and PTSD. PTSD } \\
\text { such as flashbacks } \\
\text { and hyper } \\
\text { vigilance around } \\
\text { places and } \\
\text { occasions that } \\
\text { reminded them } \\
\text { of their sexual } \\
\text { abuse were } \\
\text { common. A few } \\
\text { women stated } \\
\text { that their distress } \\
\text { led to frequent } \\
\text { visits to the } \\
\text { hospital for } \\
\text { physical complaints } \\
\text { as they } \\
\text { psychologically } \\
\text { struggled to } \\
\text { comprehend } \\
\text { their sexually } \\
\text { abusive } \\
\text { experiences since }\end{array}$ & $\begin{array}{l}75 \% \text { reported } \\
\text { sexual } \\
\text { revictimization. }\end{array}$ \\
\hline
\end{tabular}




\begin{tabular}{|c|c|c|c|c|c|c|c|c|c|}
\hline & & & & & & & & $\begin{array}{l}\text { childhood. Using } \\
\text { illicit substances } \\
\text { (e.g. drugs) } \\
\text { helped all the } \\
\text { women numb } \\
\text { their symptoms } \\
\text { of emotional } \\
\text { distress and } \\
\text { feelings of anger } \\
\text { and betrayal } \\
\text { generated by } \\
\text { their CSA. }\end{array}$ & \\
\hline $\begin{array}{l}\text { (27) Tarakeshwar } \\
\text { (2006) [51] }\end{array}$ & $\begin{array}{l}266 \\
(266 / 0)\end{array}$ & $\begin{array}{l}\text { USA. } 266 \\
\text { HIV-positive } \\
\text { participants. } \\
\text { There were } \\
133 \text { males, } \\
129 \text { females, } \\
\text { and } 4 \\
\text { transgender. } \\
\text { The majority } \\
(71.5 \%) \text { was } \\
\text { African- } \\
\text { American. }\end{array}$ & $\begin{array}{l}\text { Survey } \\
\text { HIV + only }\end{array}$ & $\begin{array}{l}\text { Quantitative } \\
\text { cross-sectional } \\
\text { survey } \\
\text { design. }\end{array}$ & $\begin{array}{l}\text { Participants were } \\
\text { screened for } \\
\text { abuse histories in } \\
\text { childhood, } \\
\text { adolescence, and } \\
\text { adulthood. The } \\
\text { BDI was used to } \\
\text { assess depressive } \\
\text { symptomatology. } \\
\text { Perspectives on } \\
\text { addressing trauma } \\
\text { symptoms, } \\
\text { HIV-related } \\
\text { stress, and } \\
\text { resiliency } \\
\text { were also } \\
\text { assessed } \\
\text { using self- } \\
\text { developed } \\
\text { screening } \\
\text { tools and } \\
\text { modified } \\
\text { scales. }\end{array}$ & $\begin{array}{l}\text { (1) Substance } \\
\text { use treatment } \\
\text { in the past } \\
\text { four months. } \\
\text { (2) Lower } \\
\text { resiliency } \\
\text { and greater } \\
\text { HIV-related } \\
\text { stress was } \\
\text { related to } \\
\text { negative } \\
\text { feelings about } \\
\text { addressing } \\
\text { trauma. } \\
\text { (3) Revictimization. }\end{array}$ & $\begin{array}{l}91 \% \text { of the } \\
\text { participants had } \\
\text { been sexually } \\
\text { abused as } \\
\text { children, } \\
77 \% \text { had been } \\
\text { abused during } \\
\text { adolescence. } \\
71.5 \% \text { of men } \\
\text { and } 66.7 \% \\
\text { of women } \\
\text { reported } \\
\text { unwanted } \\
\text { vaginal } \\
\text { or anal sex in } \\
\text { childhood. }\end{array}$ & $\begin{array}{l}54 \% \text { of men } \\
\text { and } 52 \% \text { of } \\
\text { women had at } \\
\text { least one visit } \\
\text { to a mental } \\
\text { health provider } \\
\text { in past } 4 \text { months. } \\
39.5 \% \text { of men } \\
\text { and } 38 \% \text { of } \\
\text { women were on } \\
\text { psychiatric } \\
\text { medications. } \\
\text { Substance use } \\
\text { treatment in the } \\
\text { past } 4 \text { months } \\
\text { was reported in } \\
38.8 \% \text { of men } \\
\text { and } 29.5 \% \text { of } \\
\text { women. }\end{array}$ & $\begin{array}{l}56 \% \text { had been } \\
\text { sexually } \\
\text { revictimized } \\
\text { as adults. }\end{array}$ \\
\hline $\begin{array}{l}\text { (28) Welles } \\
\text { (2009) [30] }\end{array}$ & $\begin{array}{l}593 \\
(593 / 0)\end{array}$ & $\begin{array}{l}\text { USA. } 593 \\
\text { HIV + MSM. }\end{array}$ & $\begin{array}{l}\text { Survey } \\
\text { HIV + only }\end{array}$ & $\begin{array}{l}\text { Baseline } \\
\text { survey for } \\
\text { a risk } \\
\text { reduction } \\
\text { intervention } \\
\text { trial. }\end{array}$ & $\begin{array}{l}\text { Participants reported } \\
\text { the frequency } \\
\text { of CSA. } \\
\text { Brief Symptom } \\
\text { Checklist } \\
\text { was used to } \\
\text { assess depression } \\
\text { and anxiety. }\end{array}$ & $\begin{array}{l}\text { (1) High levels of } \\
\text { depression. } \\
\text { (2) High levels } \\
\text { of anxiety. } \\
\text { (3) Reported } \\
\text { current } \\
\text { and lifetime } \\
\text { alcohol and } \\
\text { drug problems. }\end{array}$ & $\begin{array}{l}\text { Of participants, } \\
47 \% \text { reported } \\
\text { CSA, with 32\% } \\
\text { reporting } \\
\text { CSA occurring } \\
\text { often or } \\
\text { sometimes. } \\
\text { Although most } \\
\text { (154 or 58\%) } \\
\text { reported the } \\
\text { gender of the } \\
\text { perpetrator as } \\
\text { male, } 38 \text { (14\%) } \\
\text { reported CSA } \\
\text { by a female, }\end{array}$ & $\begin{array}{l}\text { HIV + reporting } \\
\text { history of CSA } \\
\text { had significantly } \\
\text { higher levels of } \\
\text { depression and } \\
\text { anxiety, with } \\
39 \% \text { reporting } \\
\text { the highest } \\
\text { quartile scores } \\
\text { for the } \\
\text { depression } \\
\text { and anxiety } \\
\text { inventory, } \\
\text { compared with } \\
24 \% \text { of men }\end{array}$ & \\
\hline
\end{tabular}


Table 1 Summary of $\mathbf{3 4}$ articles selected for review (Continued)

\begin{tabular}{|c|c|c|c|c|c|c|c|c|c|}
\hline & & & & & & & $\begin{array}{l}\text { and } 75(28 \%) \\
\text { by both. }\end{array}$ & $\begin{array}{l}\text { reporting no } \\
\text { CSA. Men } \\
\text { reporting CSA } \\
\text { were more likely } \\
\text { to believe that } \\
\text { they have or } \\
\text { had problems } \\
\text { with drugs or } \\
\text { alcohol. }\end{array}$ & \\
\hline $\begin{array}{l}\text { (29) Wyatt } \\
\text { (2005) [52] }\end{array}$ & $\begin{array}{l}75 \\
(75 / 0) .\end{array}$ & $\begin{array}{l}\text { USA. } 75 \\
\text { HIV-positive } \\
\text { women with } \\
\text { histories } \\
\text { of CSA. }\end{array}$ & $\begin{array}{l}\text { Survey } \\
\text { HIV + only }\end{array}$ & $\begin{array}{l}\text { Baseline } \\
\text { survey for } \\
\text { an intervention } \\
\text { trial. }\end{array}$ & $\begin{array}{l}\text { Women were } \\
\text { administered the } \\
\text { WSHQ-R. Five } \\
\text { measures were } \\
\text { used to assess } \\
\text { patterns of } \\
\text { substance } \\
\text { abuse. }\end{array}$ & $\begin{array}{l}\text { (1) Substance } \\
\text { abuse. } \\
\text { (2) Lifetime } \\
\text { alcohol } \\
\text { or drug } \\
\text { treatment. }\end{array}$ & $\begin{array}{l}\text { All women in the } \\
\text { sample had a } \\
\text { history of CSA. }\end{array}$ & $\begin{array}{l}83 \% \text { of the } \\
\text { sample reported } \\
\text { having used at } \\
\text { least } 1 \text { of } 13 \\
\text { substances } \\
\text { regularly at } \\
\text { some point in } \\
\text { their lives. } 28 \% \\
\text { of the sample } \\
\text { reported engaging } \\
\text { in regular injection } \\
\text { drug use. } 54 \% \\
\text { of the women } \\
\text { reported having } \\
\text { taken part in an } \\
\text { alcohol or drug } \\
\text { treatment } \\
\text { program at } \\
\text { some point in } \\
\text { their lifetime. }\end{array}$ & \\
\hline $\begin{array}{l}\text { (30) Paxton } \\
\text { (2004) [53] }\end{array}$ & $\begin{array}{l}457 \\
(299 / \\
158)\end{array}$ & $\begin{array}{l}\text { USA. } 65.4 \% \\
\text { of the sample } \\
\text { was HIV- } \\
\text { seropositive. } \\
\text { The majority } \\
\text { of the sample } \\
\text { was African- } \\
\text { American. }\end{array}$ & $\begin{array}{l}\text { General } \\
\text { survey } \\
\text { (mixed). }\end{array}$ & $\begin{array}{l}\text { Quantitative } \\
\text { cross-sectional } \\
\text { survey } \\
\text { design. }\end{array}$ & $\begin{array}{l}\text { Alcohol and } \\
\text { drug abuse/ } \\
\text { dependence, } \\
\text { depression, and } \\
\text { panic disorder: } \\
\text { subscales of } \\
\text { the University } \\
\text { of Michigan } \\
\text { Revised Short } \\
\text { Form of } \\
\text { the Composite } \\
\text { International } \\
\text { Diagnostic } \\
\text { Inventory. } \\
\text { Posttraumatic } \\
\text { stress symptoms: } \\
\text { revised }\end{array}$ & $\begin{array}{l}\text { (1) PTSD } \\
\text { symptoms. } \\
\text { (2) Substance } \\
\text { abuse. } \\
\text { (3) Risky health } \\
\text { behaviors. } \\
\text { (4) Chronic } \\
\text { stress. } \\
\text { (5) History of } \\
\text { psychiatric } \\
\text { disorders. }\end{array}$ & $\begin{array}{l}\text { HIV-positive } \\
\text { women were } \\
\text { more likely to } \\
\text { report a history } \\
\text { of CSA. }\end{array}$ & $\begin{array}{l}\text { HIV-positive } \\
\text { women with } \\
\text { a history of } \\
\text { CSA were } \\
\text { more likely } \\
\text { to report } \\
\text { posttraumatic } \\
\text { stress, substance } \\
\text { abuse, } \\
\text { chronic stress, } \\
\text { and psychiatric } \\
\text { history than } \\
\text { HIV-negative } \\
\text { counterparts. }\end{array}$ & $\begin{array}{l}\text { HIV-positive } \\
\text { women with } \\
\text { a history of } \\
\text { CSA were } \\
\text { more likely } \\
\text { to report } \\
\text { risky health } \\
\text { behaviors } \\
\text { than HIV- } \\
\text { negative } \\
\text { counterparts. }\end{array}$ \\
\hline
\end{tabular}


Table 1 Summary of $\mathbf{3 4}$ articles selected for review (Continued)

17-item short

form clinical

checklist.

Select items

from the

WSHQ-R measured

exposure to

sexual and

trauma.

\begin{tabular}{|c|c|c|c|c|c|c|c|c|c|}
\hline $\begin{array}{l}\text { (31) Cohen } \\
\text { (2000) [6] }\end{array}$ & $\begin{array}{l}1645 \\
(1288 / \\
357) .\end{array}$ & $\begin{array}{l}\text { USA. } 1288 \\
\text { HIV-positive } \\
\text { women and } \\
357 \text { HIV-negative } \\
\text { women. The } \\
\text { majority (64\%) } \\
\text { were African- } \\
\text { American. }\end{array}$ & $\begin{array}{l}\text { General } \\
\text { survey } \\
\text { (mixed). }\end{array}$ & $\begin{array}{l}\text { Quantitative } \\
\text { cross-sectional } \\
\text { survey design: } \\
\text { retrospective } \\
\text { data. }\end{array}$ & $\begin{array}{l}\text { A survey } \\
\text { investigating } \\
\text { three areas of } \\
\text { violence: } \\
\text { any domestic } \\
\text { violence, } \\
\text { recent domestic } \\
\text { violence } \\
\text { and CSA. Lifetime } \\
\text { substance } \\
\text { abuse and } \\
\text { injection drug } \\
\text { use in the past } \\
6 \text { months was } \\
\text { assessed. Finally, } \\
\text { HIV risk behaviors } \\
\text { were assessed. }\end{array}$ & $\begin{array}{l}\text { (1) Drug use. } \\
\text { (2) HIV-risk } \\
\text { behaviors. } \\
\text { (3) Revictimization. }\end{array}$ & $\begin{array}{l}31 \% \text { of HIV- } \\
\text { positive } \\
\text { women } \\
\text { reported a } \\
\text { history } \\
\text { of CSA }\end{array}$ & $\begin{array}{l}\text { Women } \\
\text { reporting } \\
\text { past domestic } \\
\text { violence or } \\
\text { CSA were } \\
\text { more likely } \\
\text { than women } \\
\text { without such } \\
\text { histories } \\
\text { to have } \\
\text { used drugs } \\
\text { at some } \\
\text { point in } \\
\text { their lives. }\end{array}$ & $\begin{array}{l}\text { Women } \\
\text { reporting } \\
\text { past domestic } \\
\text { violence or } \\
\text { CSA were } \\
\text { more likely } \\
\text { than women } \\
\text { without such } \\
\text { histories to } \\
\text { have had more } \\
\text { than } 10 \text { lifetime } \\
\text { male partners; } \\
\text { to have traded } \\
\text { sex for money, } \\
\text { drugs, or shelter; } \\
\text { and to have } \\
\text { been forced to } \\
\text { have sex with a } \\
\text { person known } \\
\text { to be HIV } \\
\text { positive. Women } \\
\text { who reported } \\
\text { CSA were more } \\
\text { likely to report } \\
\text { a lifetime history } \\
\text { of domestic } \\
\text { violence and to } \\
\text { have experienced } \\
\text { domestic } \\
\text { violence in the } \\
\text { past year. }\end{array}$ \\
\hline $\begin{array}{l}\text { (32) Kalichman } \\
\left(2004^{a}\right)[3]\end{array}$ & $\begin{array}{l}272 \\
(6 / 19)\end{array}$ & $\begin{array}{l}\text { South Africa. } \\
272 \text { women } \\
\text { living with } \\
\text { sexual assault } \\
\text { histories. Nearly } \\
\text { all (99\%) } \\
\text { of the women } \\
\text { were African. }\end{array}$ & $\begin{array}{l}\text { General } \\
\text { survey } \\
\text { (mixed). }\end{array}$ & $\begin{array}{l}\text { Quantitative } \\
\text { cross-sectional } \\
\text { survey } \\
\text { design. }\end{array}$ & $\begin{array}{l}\text { Self-administered } \\
\text { anonymous } \\
\text { surveys } \\
\text { assessing sexual } \\
\text { assault history, } \\
\text { substance use, } \\
\text { history of HIV } \\
\text { risk factors, }\end{array}$ & $\begin{array}{l}\text { (1) Alcohol and } \\
\text { drug use. } \\
\text { (2) Transactional } \\
\text { sex }\end{array}$ & $\begin{array}{l}6 \text { women (11\%) } \\
\text { were HIV-positive } \\
\text { and } 19(33 \%) \text { were } \\
\text { HIV-negative. The } \\
\text { majority of women } \\
\text { (56\%) did not } \\
\text { know their } \\
\text { HIV status. } 40 \% \\
\text { ( } N=/ 119) \text { of }\end{array}$ & & $\begin{array}{l}\text { Women who } \\
\text { had been } \\
\text { sexually } \\
\text { assaulted were } \\
\text { significantly } \\
\text { more likely to } \\
\text { have shared } \\
\text { injection drug } \\
\text { equipment, }\end{array}$ \\
\hline
\end{tabular}




\begin{tabular}{|c|c|c|c|c|c|c|c|c|c|}
\hline & & & & & $\begin{array}{l}\text { and sexual } \\
\text { behavior. }\end{array}$ & & $\begin{array}{l}\text { women reported } \\
\text { a history of } \\
\text { sexual assault. } \\
26(21 \%) \\
\text { of the women } \\
\text { had experienced } \\
\text { sexual assault } \\
\text { before the } \\
\text { age of } 20 .\end{array}$ & & $\begin{array}{l}\text { exchanged sex } \\
\text { to meet survival } \\
\text { needs, and } \\
\text { used alcohol } \\
\text { compared to } \\
\text { women who } \\
\text { had not been } \\
\text { sexually } \\
\text { assaulted. }\end{array}$ \\
\hline $\begin{array}{l}\text { (33) Kalichman } \\
\left(2004^{b}\right)[54]\end{array}$ & $\begin{array}{l}647 \\
(498 / \\
142) .\end{array}$ & $\begin{array}{l}\text { USA. } 647 \text { men } \\
\text { with CSA } \\
\text { histories. } \\
\text { The majority } \\
\text { were Caucasian } \\
(70 \%) \text {. }\end{array}$ & $\begin{array}{l}\text { General } \\
\text { survey } \\
\text { (mixed). }\end{array}$ & $\begin{array}{l}\text { Quantitative } \\
\text { cross-sectional } \\
\text { survey } \\
\text { design. }\end{array}$ & $\begin{array}{l}\text { Self-administered } \\
\text { surveys were used } \\
\text { to assess } \\
\text { demographics, } \\
\text { sexual abuse } \\
\text { history, } \\
\text { substance use } \\
\text { and sexual } \\
\text { risk behaviors. }\end{array}$ & $\begin{array}{l}\text { (1) Symptoms of } \\
\text { borderline } \\
\text { personality } \\
\text { disorder. } \\
\text { (2) Alcohol and } \\
\text { drug abuse. } \\
\text { (3) Having } \\
\text { undergone } \\
\text { treatment for } \\
\text { substance } \\
\text { abuse. }\end{array}$ & $\begin{array}{l}93(15 \%) \text { of } \\
\text { the men } \\
\text { reported being } \\
\text { forced to have } \\
\text { sex when they } \\
\text { were } 16 \text { years or } \\
\text { younger by a man } \\
\text { at least } 5 \text { years } \\
\text { older. Of these } \\
93 \text { men, the } \\
\text { average age of first } \\
\text { abuse was } 9.3 \\
\text { years. Sexually } \\
\text { abused men were } \\
\text { more likely to } \\
\text { report childhood } \\
\text { physical abuse } \\
\text { relative to non- } \\
\text { abused men (41\% } \\
\text { vs. 12\%). Men } \\
\text { who were sexually } \\
\text { abuse were more } \\
\text { likely to have } \\
\text { tested HIV-positive } \\
\text { (40\%) relative to } \\
\text { non-abused men } \\
\text { (19\%). } 77 \% \text { of } \\
\text { the men were } \\
\text { HIV-negative and } \\
22 \% \text { were (9\%). }\end{array}$ & $\begin{array}{l}\text { HIV-positive. } \\
\text { Abused } \\
\text { men endorsed } \\
\text { more symptoms } \\
\text { of borderline } \\
\text { personality } \\
\text { disorder. Contrary } \\
\text { to expectations, } \\
\text { abused men } \\
\text { did not differ in } \\
\text { dissociation } \\
\text { symptoms or } \\
\text { trauma-related } \\
\text { anxiety when } \\
\text { compared to } \\
\text { non-abused } \\
\text { counterparts. } \\
\text { Abused men } \\
\text { were more } \\
\text { likely to report } \\
\text { alcohol and drug } \\
\text { abuse in the past } \\
6 \text { months and } \\
\text { having undergone } \\
\text { treatment (28\%) } \\
\text { compared to } \\
\text { non-abused men. }\end{array}$ & \\
\hline $\begin{array}{l}(34) \text { Kimerling } \\
\left(1999^{b}\right)[27]\end{array}$ & $\begin{array}{l}236 \\
(88 / \\
148)\end{array}$ & $\begin{array}{l}\text { USA. } 88 \text { African- } \\
\text { American } \\
\text { HIV-infected } \\
\text { women } \\
\text { and } 148 \\
\text { uninfected } \\
\text { women. }\end{array}$ & $\begin{array}{l}\text { General } \\
\text { survey } \\
\text { (mixed). }\end{array}$ & $\begin{array}{l}\text { Quantitative } \\
\text { cross-sectional } \\
\text { survey design: } \\
\text { retrospective } \\
\text { data. }\end{array}$ & $\begin{array}{l}\text { The Life Stressor } \\
\text { Checklist: history } \\
\text { of victimization. } \\
\text { The BSI: level of } \\
\text { general or global } \\
\text { distress. The } \\
\text { Hamilton Clinician's } \\
\text { Rating Scale for } \\
\text { Depression: } \\
\text { depression }\end{array}$ & $\begin{array}{l}\text { (1) High levels } \\
\text { of global } \\
\text { psychological } \\
\text { distress. } \\
\text { (2) Depression. } \\
\text { (3) Greater } \\
\text { physical } \\
\text { distress and } \\
\text { AIDS- }\end{array}$ & $\begin{array}{l}\text { A history of } \\
\text { completed rape } \\
\text { contributed the } \\
\text { greatest risk for } \\
\text { HIV infection. } \\
\text { Women who } \\
\text { reported } \\
\text { completed rape } \\
\text { identified the } \\
\text { worst experience }\end{array}$ & $\begin{array}{l}\text { HIV-infected } \\
\text { victims reported } \\
\text { higher levels of } \\
\text { global } \\
\text { psychological } \\
\text { distress, and } \\
\text { greater severity } \\
\text { of clinician-rated } \\
\text { symptoms of } \\
\text { depression. HIV- }\end{array}$ & \\
\hline
\end{tabular}


Table 1 Summary of $\mathbf{3 4}$ articles selected for review (Continued)

$\begin{array}{llll}\text { and to serve as } & \text { defining } & \text { to have occurred } & \text { infected victims } \\ \text { a more objective } & \text { conditions. } & \text { at 18.27 years } & \text { also reported } \\ \text { measure of } & & \text { old. This variable } & \text { significantly } \\ \text { psychological } & \text { included rape } & \text { greater distress } \\ \text { distress. } & \text { as a child. } & \text { with physical } \\ & & \text { symptoms and } \\ & & \text { higher rates of } \\ & & \text { AIDS-defining } \\ & \text { conditions than } \\ & \text { did non-victims. }\end{array}$

BDI, Beck Depression Inventory; BSI, Brief Symptom Inventory; CES-D, Center for Epidemiologic Studies Depression Scale; CSA, childhood sexual abuse; CTQ, Childhood Trauma Questionnaire; EPQ, Eysenck Personality $B D I$, Beck Depression Inventory; BSI, Brief Symptom Inventory; CES-D, Center for Epidemiologic Studies Depression Scale; $C S A$, childhood sexual abuse; CTQ, Childhood Trauma Questionnaire; EPQ, Eysenck Personality
Questionnaire; HAART, highly active antiretroviral therapy; IVDU, intravenous drug use; MDD, Major depressive disorder; MSM, men who have sex with men; PTSD, posttraumatic stress disorder; PSUD, Psychoactive Questionnaire; HAART, highly active antiretroviral therapy; IVDU, intravenous drug use; MDD, Major depressive disorder; MSM, men who have sex with men; PTSD, posttraumatic stress disorder; PSUD, Psychoactive
Substance Use Disorder; SNAP, schedule for nonadaptive personality; STI, sexually transmitted infection; TEQ, Traumatic Experiences Questionnaire; TSI, Trauma Symptom Inventory; WSHQ-R, revised Wyatt Sex History Substance Use Questionnaire. 
Trauma Questionnaire [4]. However, many studies established a history of childhood abuse by simply asking a single question such as have you ever experienced a sexual assault or rape as a child or teenager, that is, when you were 18 years of age or younger?' and using a dichotomous response option (Yes/No) [26,28,42].

\section{Childhood maltreatment}

Childhood maltreatment, such as physical and sexual abuse is a common phenomenon in the general population (uninfected individuals). CSA is reported by as many as $32 \%$ of women and $14 \%$ of men in the general population, whereas physical abuse is experienced by $22 \%$ of males and $19.5 \%$ of females in the general population [55]. However, rates of childhood maltreatment in HIV-positive individuals are significantly higher, suggesting that the experience of childhood maltreatment in the context of HIV is worthy of greater attention. Rates of CSA among HIV-positive individuals range from 32\% to $76 \%$, respectively $[28,56,57]$.

\section{Mental health outcomes}

In reviewing the articles, a wide range of mental health symptoms and disorders were reported. The most commonly reported psychiatric symptomatology among HIV-positive individuals with a history of childhood maltreatment included (study number in Table 1): drug and/or alcohol abuse/dependence (2,4,7,9-17,20-24,26-33), depression $(2,4,6,9,10,13,15,17,20-26,28)$ and posttraumatic stress disorder (PTSD) (1,3-5,8,12,18-20,23,24,26,30). Other mental health outcomes reported included (reference number in Table 1$)$ : anxiety $(4,16,22-24,26,28)$, generalized anxiety disorder (20), borderline personality $(16,33)$, panic disorder $(21,26)$, agoraphobia (26), schizophrenia (21), psychotic disorder (22), adjustment disorder (22), bipolar disorder (22), suicidality $(9,21)$, neuroticism (9), personality disorder (4) and multiple personality disorder (21). Moreover, when examining mental health outcomes such as drug abuse and depressive symptomatology, two articles also reported an association between childhood maltreatment and poor treatment adherence to antiretroviral regimens $(13,17)$. Physical complaints/distress and reduced quality of life was also a finding in the studies reviewed $(14,18,26)$. Findings from several studies indicated that participants had at some time in their lives undergone mental health treatment (22,26,27,29,33). Many studies found participants commonly reporting engagement in high-risk behaviors such as transactional sex or compulsive sexual behaviors $(10,11,15,21,30-32)$ and adult revictimization was common $(3,4,10,24,26,27,31)$. Individual rates of psychopathology reported in studies varied. The percentage of participants in individual studies who received a diagnosis of PTSD included (study number in Table 1): 42.9\% (1), 40\% (3), 32\% (4), 40\% (5),
35\% (18), 42\% (19), 28\% (20) and 16\% (23). Other studies reported PTSD scores on self-report/interviewer administered instruments. The average PTSD score on the Posttraumatic Diagnostic Scale was 20.75 in one study, with $30 \%$ of participants reporting moderate to severe symptoms and 15\% reporting severe symptoms (12). In another study, the mean score on the Trauma Symptom Inventory for trauma-related symptoms was 40.4 in women and 28.9 in men (24). PTSD was not an inclusion criterion in the research study but rather an unselected observation for most studies $(1,3,5,12,20,23,24)$. However, in one study, participants were only included if there was evidence of psychological distress or if criteria for mood or anxiety disorders were met (4).

The percentage of participants in individual studies who received a diagnosis of mood disorders included (study number in Table 1): 46.4\% (4) 68\% (10), 15\% (20), $30 \%$ (22), 34.7\% (23) and 39\% (28). Other studies reported depression scores on self-report/interviewer administered instruments. The mean depression score on the Center for Epidemiologic Studies Depression Scale (CES-D) was 23 in one study (6) and in another study, $81 \%$ of women and $76 \%$ of men had a depression score higher than 16 on the CES-D (17). The mean score for depressive symptoms on the Beck Depression Inventory was 29.8 in women and 28.2 in men in another study (24). Mood disorders was not an inclusion criterion in the research study but rather an unselected observation for most studies $(20,23,24,28)$. However, in other studies, participants were only included if there was evidence of psychological distress or criteria for mood or anxiety disorders were met $(4,22)$.

The percentage of participants in individual studies who received a diagnosis of drug and/or alcohol dependence/abuse included (study number in Table 1): 25\% (4), $58 \%$ lifetime and 9\% current (7) 77\% (9), 88\% (10), 55\% (14), $19 \%$ and $31 \%(20), 37 \%$ and $10 \%(22), 20 \%(23)$, $31.8 \%, 53.2 \%, 18.2 \%, 36.3 \%, 33.1 \%$ and $18.9 \%$ (24), $38.3 \%$ (27), 28\% (29) and 28\% (33). Other studies did not report individual rates but suggested that abused HIVpositive individuals were more likely to have engaged in alcohol or drug abuse and received treatment for substance abuse (2,11-13,15-17,21,26,28,30-32). Drug and/ or alcohol dependence/abuse was not an inclusion criteria in the research study but rather an unselected observation for most studies (2,4,7,11-16,20-24,26-33). However, in other studies, participants were only included if there was evidence of drug and/or alcohol dependence/abuse $(9,17)$.

In comparison to the general population (i.e. uninfected and non-abused counterparts), evidence suggests that ongoing risk behaviors and rates of psychopathology are higher in HIV-infected individuals with histories of abuse $[7,8,10,31,37,53]$. HIV-positive individuals were 
more likely to report posttraumatic stress, risky health behaviors, substance abuse, chronic stress, and psychiatric history compared with HIV-negative counterparts [53]. In addition, abused individuals reported higher rates of mental illness, compared to non-abused counterparts, suggesting that a history of abuse in childhood increases the likelihood of psychopathology [58]. These findings lend credence to the argument that childhood maltreatment in the context of HIV is worthy of greater attention.

\section{Intervention studies}

The review revealed six intervention studies that have been conducted with this population [8,31-35]. Three of these interventions were carried out in bisexual men and men who have sex with men (MSM) $[8,31,35]$ and three were carried out in mixed samples of males and females [32-34]. A total of 4295 MSM were enrolled into a behavioral intervention trial over 48 months. Behavioral assessments were conducted every 6 months. However, the results revealed that among men reporting a history of CSA, the intervention had no effect in reducing HIV infection rates. Moreover, men reporting a history of CSA were more likely to display depressive symptomatology and use nonprescription drugs [8]. Similarly, 49 gay and bisexual HIV-infected men with histories of CSA were enrolled into an intervention study, consisting of 15 coping group sessions. When compared to an alternative support group intervention and a control condition, the coping group intervention proved to be efficacious in treating HIV-positive adults with histories of CSA. This was attributable to the inclusion of a coping skills training component in the aforementioned treatment condition [31]. Support for the efficacy of the aforementioned coping group intervention was reported in a separate study assessing 28 men and women with HIV and histories of CSA [33]. Similarly findings were reported in another study utilizing the same coping group intervention in 198 HIV-infected men and women with histories of CSA [34]. Reductions in intrusive traumatic stress symptoms were exhibited among participants in the coping group intervention compared to the waitlist condition and in avoidant traumatic stress symptoms compared to the support group condition [34]. Moreover, the efficacy of the aforementioned coping group intervention in reducing sexual transmission risk behavior was assessed [32]. The sexual behavior of 247 HIVpositive men and women with histories of CSA was assessed at baseline, postintervention, and at 4, 8, and 12 month follow-up periods. The frequency of unprotected sexual intercourse for all partners decreased more among participants in the coping group intervention than participants in the support intervention condition [32]. Lastly, a randomized clinical trial comparing the effects of two six-session interventions was carried out in a sample of 137 bisexual men and MSM. Results from both interventions revealed reductions in sexual risk behaviors and number of sexual partners from baseline to posttest, and from 3 to 6 month follow-ups. No significant differences in depression were evident between the two conditions; however, at 6 months the total sample reported a significant decrease in depressive symptoms [35].

\section{Adherence to antiretroviral medication}

In examining mental health outcomes, two articles also reported an association between childhood maltreatment and poor treatment adherence to antiretroviral regimens $[25,43]$. In one study, a lower percentage of women with a history of physical or sexual abuse reported using Highly Active Antiretroviral Therapies (HAART). Experiencing any physical or sexual abuse increased the likelihood of no HAART use. Women with a history of any physical or sexual abuse were more than 1.5 times more likely to lack HAART, even when clinically eligible [25]. Moreover, the use of HIV medications has been found to be negatively associated with CSA experiences [43].

\section{High risk behaviors}

Many studies found participants commonly reporting engagement in high-risk behaviors such as transactional sex or compulsive sexual behaviors $[3,6,39,40,42,53]$. Individuals who experienced abuse regularly were more likely to be HIV-positive, exchanged sex for payment, and be a current user of sex-related drugs [40]. It has also been reported that women experiencing CSA were more likely than women without such histories to have used drugs, to have had more than ten sexual partners, to have traded sex for money, drugs, or shelter; and to have been forced to have sex with a person known to be HIV-positive [6]. Moreover, women who had been sexually assaulted were significantly more likely to have shared injection drug equipment [3].

\section{Discussion}

We performed a comprehensive systematic review of the literature to assess mental health outcomes in HIVpositive individuals with histories of childhood maltreatment. To our knowledge, this is the first review of its kind; no published systematic reviews assessing this association have been conducted to date.

The reported mental health outcomes in dually affected individuals (HIV-positive individuals with histories of childhood maltreatment) are in keeping with studies that have investigated these variables separately [11-19,23], supporting at least common outcomes, 
although assessment of the additive effects of HIV and childhood trauma is difficult in this retrospective review.

The most commonly reported mental illnesses in dually affected individuals included mood, anxiety, and substance abuse disorders. Very few studies examined Axis II disorders. It has been suggested that an HIV diagnosis alone may constitute a significant stressor and thus increase the likelihood of mental illnesses among HIV-positive individuals [59]. Apart from depression or anxiety being a secondary diagnosis to HIV/AIDS, anxiety and depressive symptoms measured over time were also associated with faster progression of the disease after five years. This finding may suggest a reinforcing relationship between HIV and mental illnesses such as depression or anxiety [20]. However, the majority of studies reviewed were cross-sectional in nature, therefore limiting their ability to make causal conclusions around the onset of mental illness in HIV. This highlights the importance of longer term assessment in order to better delineate the nature, severity, and temporal nature of mental health outcomes. Importantly, mental disorders such as depression or anxiety can further impact immune system functioning in HIV and, in turn, influence quality of life and health status [60].

Substance abuse was the most predominant mental health outcome reported in reviewed articles. For the most part, drugs and/or alcohol are used to numb emotional distress and feelings of anger and betrayal resulting from the experience of childhood maltreatment [50]. Not only does substance abuse have direct implications for the progression of the disease in infected individuals [61], it also has direct and indirect implications for the transmission of HIV. Antiretroviral regimens are known to have strong positive effects on quality of life and in improving health status in infected individuals [62]. Few articles have reported an association between childhood maltreatment and poor treatment adherence to antiretroviral regimens or HIV medications $[25,43]$.

Some studies that investigated both early life trauma and adult trauma found an association between childhood trauma and later life trauma [6,28,32,37]. For example, the study by Simoni and $\mathrm{Ng}$ found that childhood abuse was correlated with both adult and recent trauma. Moreover, each type of trauma was also correlated with depression scores [49]. Several studies have also found that adult revictimization was very common in survivors of childhood maltreatment $[6,32,33,39,50,51]$. Further investigation of this relationship and the implications for prevention and intervention is warranted.

HIV-infected men and women may face many current and past negative life events [13] and this may lead to significant adult psychopathology and poor adherence to antiretroviral medications [24,25,63]. In light of this, it is evident that HIV-positive individuals, women in particular, are vulnerable to risk factors associated with abuse, and abuse-related changes in behavioral functioning. These risk factors and behavioral changes may in turn complicate HIV infection.

There are several limitations that warrant mention. First, no search strategy can guarantee the identification of all relevant research, and omission of important studies remains a possibility and may contribute to bias in inferences drawn. Selection or reviewer bias may be a possibility given that studies were not screened or abstracted in duplicate. Second, the heterogeneity across studies presents a problem as it impedes statistical pooling of studies. Third, it is important to note the controversy that abounds in the classification of search terms. There is no standard definition for the experience of abuse as a child. This construct is one that is classified and assessed in a variety of ways. Some studies use broader terms such as childhood adversity or maltreatment, whereas others use more specific terms such as child abuse. For example, in some studies reviewed, childhood maltreatment included diverse types of trauma such as physical abuse and neglect, emotional abuse and neglect, and sexual abuse. Other studies have used more restricted definitions and have only examined CSA or resorted to combining sexual and physical abuse into one category of child abuse. Childhood maltreatment in the present review included emotional, physical, and sexual abuse and neglect. The lack of a standard definition is a further source of bias. Another limitation that warrants mention is that of the design of the review process. The selection criterion for the inclusion of studies in the present review was manuscripts reporting on mental health and childhood maltreatment. However, this should not be mistaken or presented as an argument that childhood maltreatment and impairments in mental health are associated. However, despite these limitations, this review adds substantially to available evidence for both clinical and research decision making.

\section{Implications for future studies}

From the present review, it is clear that very few prospective studies have been executed in this domain $[31,44]$. The majority of research has been crosssectional and has included retrospective assessment of childhood maltreatment in HIV-infected individuals. This may be partly due to reasons associated with feasibility and logistics. As cross-sectional study designs preclude follow-up observations and longer term assessment of outcomes, future research should be prospective in nature and should better delineate the nature, severity, and temporal relationship of childhood maltreatment to 
mental health outcomes and treatment utilization, as well as the mediators and moderators of these outcomes. These studies will allow both clinicians and researchers to better understand the etiology of common mental disorders in HIV-infected samples and reduce bias when making causal inferences. Thus, longitudinal investigation of mental health outcomes in HIV infected individuals with childhood maltreatment will be key to explaining these causal relationships.

\section{Conclusion}

A broad range of adult psychopathology has been reported in studies of HIV-infected individuals with a history of childhood maltreatment. However, a direct causal link cannot be well established. The longer term assessment will better delineate the nature, severity, and temporal relationship of childhood maltreatment to mental health outcomes. There is a need to screen for childhood maltreatment, psychopathology, and associated functioning in HIV-positive individuals and to address these issues in management. Increased focus on the identification and support for children and youth who have experienced childhood maltreatment is necessary. HIV prevention interventions such as education in high-risk behaviors are also a necessity.

\section{Competing interests}

We declare that we have no conflicts of interests.

\section{Authors' contributions}

GS performed the systematic review and drafted the manuscript. TA, SA, CF-N, JS, and SS participated in its design and coordination and helped to draft the manuscript. All authors read and approved the final manuscript.

\section{Acknowledgements}

This work is based upon research supported by the South African Research Chairs Initiative of the Department of Science and Technology and National Research Foundation; the MRC Unit on Anxiety and Stress Disorders, Department of Psychiatry, University of Stellenbosch, Cape Town, South Africa; the Claude Leon Foundation; the Hendrik Vrouwes Scholarship; the Centers for AIDS Research (CFAR); and the HIV Neurobehavioral Research Center (HNRC; National Institute of Mental Health P30-MH62512).

\section{Author details}

${ }^{1}$ South African Research Chairs Initiative (SARChI), PTSD program, Francie van Zijl drive, Department of Psychiatry, University of Stellenbosch, Cape Town 7505, South Africa. ${ }^{2}$ MRC Unit on Anxiety and Stress Disorders, Francie van Zijl drive, Department of Psychiatry, University of Stellenbosch, Cape Town 7505, South Africa. ${ }^{3}$ Departments of Psychiatry, Psychology, and Community Health Sciences, Bannatyne Avenue, University of Manitoba, Winnipeg R3E OW5, Canada. ${ }^{4}$ Department of Community Health Sciences, Bannatyne Avenue, University of Manitoba, Winnipeg R3E OW5, Canada. ${ }^{5}$ Department of Psychiatry, Gilman drive, University of California San Diego, La Jolla, CA 92093, USA. ${ }^{6}$ Department of Radiology, Gilman Drive, University of California San Diego, La Jolla, CA 92093, USA.

Received: 16 September 2011 Accepted: 16 May 2012 Published: 28 June 2012

\section{References}

1. Andersson N, Cockcroft A, Shea B: Gender-based violence and HIV: relevance for HIV prevention in hyperendemic countries of southern Africa. AIDS 2008, 22(Suppl 4):73-86.
2. Jewkes R, Penn-Kekana L, Levin J, Ratsaka M, Schrieber M: Prevalence of emotional, physical and sexual abuse of women in three South African provinces. S Afr Med J 2001, 91:421-428.

3. Kalichman SC, Simbayi LC: Sexual assault history and risks for sexually transmitted infections among women in an African township in Cape Town, South Africa. AIDS Care 2004, 16:681-689.

4. Bernstein DP, Stein JA, Newcomb MD, Walker E, Pogge D, Ahluvalia T, Stokes J, Handelsman L, Medrano M, Desmond D, Zule W: Development and validation of a brief screening version of the Childhood Trauma Questionnaire. Child Abuse Negl 2003, 27:169-190.

5. Arriola KRJ, Louden T, Doldren MA, Fortenberry RM: A meta-analysis of the relationship of child sexual abuse to HIV risk behavior among women. Child Abuse Negl 2005, 29:725-746.

6. Cohen M, Deamant C, Barkan S, Richardson J, Young M, Holman S, Anastos K, Cohen J, Melnick S: Domestic violence and childhood sexual abuse in HIV-infected women and women at risk for HIV. Am J Public Health 2000, 90:560-565.

7. Meade CS, Kershaw TS, Hansen NB, Sikkema KJ: Long-term correlates of childhood abuse among adults with severe mental illness: adult victimization, substance abuse, and HIV sexual risk behavior. AIDS Behav 2009, 13:207-216.

8. Mimiaga MJ, Noonan E, Donnell D, Safren SA, Koenen KC, Gortmaker S, O'Cleirigh C, Chesney MA, Coates TJ, Koblin BA, Mayer KH: Childhood sexual abuse is highly associated with HIV risk-taking behavior and infection among MSM in the EXPLORE Study. J Acquir Immune Defic Syndr 2009, 51:340-348.

9. Mosack KE, Randolph ME, Dickson-Gomez J, Abbott M, Smith E, Weeks MR: Sexual risk-taking among high-risk urban women with and without histories of childhood sexual abuse: mediating effects of contextual factors. J Child Sex Abus 2010, 19:43-61.

10. Wilson HW, Widom CS: An examination of risky sexual behavior and HIV in victims of child abuse and neglect: a 30-year follow-up. Health Psychol 2008, 27:149-158.

11. Johnson CF: Child sexual abuse. Lancet 2004, 364:462-470.

12. Evans DL, Ten Have TR, Douglas SD, Gettes DR, Morrison M, Chiappinni MS, Brinker-Spence P, Job C, Mercer DE, Wang YL, Cruess D, Dube B, Dalen EA, Brown T, Bauer R, Pettito JM: Association of depression with viral load, CD8 T lymphocytes, and natural killer cells in women with HIV infection. Am J Psychiatry 2002, 159:1752-1759.

13. Lipsitz JD, Williams JBW, Rabkin JG, Remien RH, Bradbury M, el Sadr W, Goetz R, Sorrell S, Gorman JM: Psychopathology in male and female intravenous drug users with and without HIV infection. Am J Psychiatry 1994, 151:1662-1668.

14. Myer L, Smit J, Roux LL, Parker S, Stein DJ, Seedat S: Common mental disorders among HIV-infected individuals in South Africa: prevalence, predictors, and validation of brief psychiatric rating scales. AIDS Patient Care STDs 2008, 22:147-158.

15. Olley Benjamin O, Gxamza Faniswa, Seedat Soraya, Theron Hugo, Taljaard Jantjie, Reid Emile, Reuter Helmuth, Stein Dan J: Psychopathology and coping in recently diagnosed HIV. S Afr Med J 2003, 93:928-931.

16. Olley BO, Seedat S, Nei DG, Stein DJ: Predictors of major depression in recently diagnosed patients with HIV/AIDS in South Africa. AIDS Patient Care STDs 2004, 18:481-487.

17. Olley BO, Zeier MD, Seedat S, Stein DJ: Post-traumatic stress disorder among recently diagnosed patients with HIV/AIDS in South Africa. AIDS Care 2005, 17:550-557.

18. Olley BO, Seedat S, Stein DJ: Persistence of psychiatric disorders in a cohort of HIV/AIDS patients in South Africa: A 6-month follow-up study. J Psychosom Res 2006, 61:479-484.

19. The WHO World Mental Health Survey Consortium: Prevalence, severity, and unmet need for treatment of mental disorders in the World Health Organization World Mental Health Surveys. JAMA 2004, 291:2581-2590.

20. Leserman J, Jackson ED, Petitto JM, Golden RN, Silva SG, Perkins DO, Cai J, Folds JD, Evans DL: Progression to AIDS: the effects of stress, depressive symptoms, and social support. Psychosom Med 1999, 61:397-406.

21. Afifi TO, Enns MW, Cox BJ, Asmundson GJ, Stein MB, Sareen J: Population attributable fractions of psychiatric disorders and suicide ideation and attempts associated with adverse childhood experiences. Am J Public Health 2008, 98:946-952. 
22. Kessler RC, McLaughlin KA, Green JG, Gruber MJ, Sampson NA, Zaslavsky AM, Aguilar-Gaxiola S, Alhamzawi AO, Alonso J, Angermeyer M, Benjet C, Bromet E, Chatterji S, de Girolamo G, Demyttenaere K, Fayyad J, Florescu S, Gal G, Gureje O, Haro JM, Hu C, Karam EG, Kawakami N, Lee S, Lepine J, Ormel J, Posada-Villa J, Sagar R, Tsang A, Ustun TB, et al: Childhood adversities and adult psychopathology in the WHO World Mental Health Surveys. Br J Psychiatry 2010, 197:378-385

23. Kaplow JB, Widom CS: Age of onset of child maltreatment predicts longterm mental health outcomes. J Abnorm Psychol 2007, 116:176-187.

24. Mugavero M, Ostermann J, Whetten K, Leserman J, Swartz M, Stangl D, Thielman N: Barriers to antiretroviral adherence: the importance of depression, abuse, and other traumatic events. AIDS Patient Care STDs 2006, 20:418-428.

25. Cohen MH, Cook JA, Grey D, Young M, Hanau LH, Tien P, Levine AM, Wilson TE: Medically eligible women who do not use HAART: the importance of abuse, drug use, and race. Am J Public Health 2004, 94:1147-1151.

26. Gielen AC, McDonnell KA, Wu AW, O'Campo P, Faden R: Quality of life among women living with HIV: the importance violence, social support and self-care behaviors. Soc Sci Med 2001, 52:315-322.

27. Kimerling R, Armistead L, Forehand R: Victimization experiences and HIV infection in women: associations with serostatus, psychological symptoms, and health status. J Trauma Stress 1999, 12:41-58.

28. Kalichman SC, Sikkema KJ, DiFonzo K, Luke W, Austin J: Emotional adjustment in survivors of sexual assault living with HIV-AIDS. J Trauma Stress 2002, 15:289-296.

29. Pence BW, Reif S, Whetten K, Leserman J, Stangl D, Swartz M, Thielman N, Mugavero MJ: Minorities, the poor, and survivors of abuse: HIV-infected patients in the US deep South. S Med J 2007, 100:1114-1122.

30. Welles SL, Baker AC, Miner MH, Brennan DJ, Jacoby S, Rosser BR: History of childhood sexual abuse and unsafe anal intercourse in a 6-city study of HIV-positive men who have sex with men. Am J Public Health 2009, 99:1079-1086.

31. Masten J, Kochman A, Hansen NB, Sikkema KJ: A short-term group treatment model for gay male survivors of childhood sexual abuse living with HIV/AIDS. Int J Group Psychother 2007, 57:475-496.

32. Sikkema KJ, Wilson PA, Hansen NB, Kochman A, Neufeld S, Ghebremichael MS, Kershaw T: Effects of a coping intervention on transmission risk behavior among people living with HIV/AIDS and a history of childhood sexual abuse. J Acquir Immune Defic Syndr 2008, 47:506-513.

33. Sikkema KJ, Hansen NB, Tarakeshwar N, Kochman A, Tate DC, Lee RS: The clinical significance of change in trauma-related symptoms following a pilot group intervention for coping with HIV-AIDS and childhood sexual trauma. AIDS Behav 2004, 8:277-291.

34. Sikkema KJ, Hansen NB, Kochman A, Tarakeshwar N, Neufeld S, Meade CS, Fox AM: Outcomes from a group intervention for coping with HIV/AIDS and childhood sexual abuse: reductions in traumatic stress. AIDS Behav 2007, 11:49-60.

35. Williams JK, Wyatt GE, Rivkin I, Ramamurthi HC, Li X, Liu H: Risk reduction for HIV-positive African American and Latino men with histories of childhood sexual abuse. Arch Sex Behav 2008, 37:763-772.

36. Holmes WC: Association between a history of childhood sexual abuse and subsequent, adolescent psychoactive substance use disorder in a sample of HIV seropositive men. J Adolesc Health 1997, 20:414-419

37. Myers HF, Wyatt GE, Loeb TB, Carmona JV, Warda U, Longshore D, Rivkin I, Chin D, Liu H: Severity of child sexual abuse, post-traumatic stress and risky sexual behaviors among HIV-positive women. AIDS Behav 2006, 10:191-199.

38. Roy A: Characteristics of HIV patients who attempt suicide. Acta Psychiatr Scand 2003, 107:41-44.

39. Allers $C T$, Benjack KJ: Connections between childhood abuse and HIV infection. J Couns Dev 1999, 70:309-313.

40. Brennan DJ, Hellerstedt WL, Ross MW, Welles SL: History of childhood sexual abuse and HIV risk behaviors in homosexual and bisexual men. Am J Public Health 2007, 97:1107-1112.

41. Clum GA, Andrinopoulos K, Muessig K, Ellen JM, Adolescent Medicine Trials Network for HIV/AIDS Interventions: Child abuse in young, HIV-positive women: linkages to risk. Qual Health Res 2009, 19:1755-1768.
42. Henny KD, Kidder DP, Stall R, Wolitski RJ: Physical and sexual abuse among homeless and unstably housed adults living with HIV: prevalence and associated risks. AIDS Behav 2007, 11:842-853

43. Kang SY, Goldstein MF, Deren S: Gender differences in health status and care among HIV-infected minority drug users. AIDS Care 2008, 20:1 146-1151.

44. Kimerling R, Calhoun KS, Forehand R, Armistead L, Morse E, Morse P, Clark R, Clark L: Traumatic stress in HIV-infected women. AIDS Educ Prev 1999, 11:321-330.

45. Martinez A, Israelski D, Walker C, Koopman C: Posttraumatic Stress Disorder in Women Attending Human Immunodeficiency Virus Outpatient Clinics. AIDS Patient Care STDs 2002, 16:283-291.

46. Martinez J, Hosek SG, Carleton RA: Screening and assessing violence and mental health disorders in a cohort of inner city HIV-positive youth between 1998-2006. AIDS Patient Care STDs 2009, 23:469-475.

47. McKeown I, Reid S, Orr P: Experiences of sexual violence and relocation in the lives of HIV infected Canadian women. Int J Circumpolar Health 2004 63(Suppl 2):399-404.

48. Sikkema KJ, Hansen NB, Meade CS, Kochman A, Fox AM: Psychosocial predictors of sexual HIV transmission risk behavior among HIV-positive adults with a sexual abuse history in childhood. Arch Sex Behav 2009, 38:121-134.

49. Simoni JM, Ng MT: Trauma, coping, and depression among women with HIV/AIDS in New York City. AIDS Care 2000, 12:567-580.

50. Tarakeshwar N, Fox A, Ferro C, Khawaja S, Kochman A, Sikkema KJ: The connections between childhood sexual abuse and Human Immunodeficiency Virus infection: implications for interventions. J Community Psychol 2005, 33:655-672.

51. Tarakeshwar N, Hansen NB, Kochman A, Fox A, Sikkema KJ: Resiliency among individuals with childhood sexual abuse and HIV: perspectives on addressing sexual trauma. J Trauma Stress 2006, 19:449-460.

52. Wyatt GE, Carmona JV, Loeb TB, Williams JK: HIV-positive black women with histories of childhood sexual abuse: patterns of substance use and barriers to health care. J Health Care Poor Underserved 2005, 16(Suppl B):9-23.

53. Paxton KC, Myers HF, Hall NM, Javanbakht M: Ethnicity, serostatus, and psychosocial differences in sexual risk behavior among HIV-seropositive and HIV-seronegative women. AIDS Behav 2004, 8:405-415.

54. Kalichman SC, Gore-Felton C, Benotsch E, Cage M, Rompa D: Trauma symptoms, sexual behaviors, and substance abuse: correlates of childhood sexual abuse and HIV risks among men who have sex with men. J Child Sex Abus 2004, 13:1-15.

55. Briere J, Elliott DM: Prevalence and psychological sequelae of selfreported childhood physical and sexual abuse in a general population sample of men and women. Child Abuse Negl 2003, 27(10):1205-1222.

56. Bedimo AL, Kissinger $P$, Bessinger R: History of sexual abuse among HIV-infected women. Int J STD AIDS 1997, 8(5):332-335.

57. Liebschutz JM, Feinman G, Sullivan L, Stein M, Samet J: Physical and sexual abuse in women infected with the human immunodeficiency virus: Increased illness and health care utilization. Arch Intern Med 2000, 160(11):1659-1664.

58. MacMillan $\mathrm{HL}$, Fleming JE, Streiner $\mathrm{DL}$, Lin E, Boyle MH, Jamieson E, Duku EK, Walsh CA, Wong MY, Beardslee WR: Childhood abuse and lifetime psychopathology in a community sample. Am J Psychiatry 2001, 158:1878-1883.

59. Nott KH, Vedhara K, Spickett GP: Psychology, immunology, and HIV. Psychoneuroendocrinology 1995, 20:451-474.

60. Cruess DG, Petitto JM, Leserman J, Douglas SD, Gettes DR, Ten Have TR, Evans DL: Depression and HIV infection: impact on immune function and disease progression. CNS Spectr 2003, 8:52-58.

61. Lucas GM, Gebo KA, Chaisson RE, Moore RD: Longitudinal assessment of the effects of drug and alcohol abuse on HIV-1 treatment outcomes in an urban clinic. AIDS 2002, 16:767-774.

62. Miller CM, Ketlhapile M, Rybasack-Smith H, Rosen S: Why are antiretroviral treatment patients lost to follow-up? A qualitative study from South Africa. Trop Med Int Health 2010, 15:48-54.

63. Pence BW: The impact of mental health and traumatic life experiences on antiretroviral treatment outcomes for people living with HIV/AIDS. J Antimicrob Chemother 2009, 63:636-640.

doi:10.1186/2046-4053-1-30

Cite this article as: Spies et al:: Mental health outcomes in HIV and childhood maltreatment: a systematic review. Systematic Reviews 2012 $1: 30$. 